\title{
Appendix
}

\section{Additional Methodology}

eTable 1 Causes of death in the Million Death Study, categorized using 3-character codes from the International Statistical Classification of Diseases and Related Health Problems version 10 (ICD-10). The estimated sensitivity and specificity of verbal autopsy is indicated for each cause of death. ${ }^{1}$

\begin{tabular}{|c|c|c|c|}
\hline Cause of Death & ICD-10 Codes & $\begin{array}{l}\text { Sensitivity } \\
(\%)\end{array}$ & $\begin{array}{l}\text { Specificity } \\
(\%)\end{array}$ \\
\hline Ischemic heart disease & I20-I25, I46, R55, R96 & 75.0 & 92.7 \\
\hline Stroke & $\begin{array}{l}\text { G45-G46, I60-I67, I69, G81- } \\
83\end{array}$ & 75.0 & 95.0 \\
\hline Injury & $\begin{array}{l}\text { D53, F79, K46, N43, Q20, } \\
\text { Q21, Q24, V02, V03, V04, } \\
\text { V05, V09, V12, V13, V14, } \\
\text { V18, V23, V24, V26, V27, } \\
\text { V28, V29, V33, V34, V38, } \\
\text { V39, V44, V47, V48, V49, } \\
\text { V54, V58, V59, V64, V68, } \\
\text { V69, V78, V79, V80, V81, } \\
\text { V84, V87, V89, V98, V99, } \\
\text { W11, W14, W15, W20, W23, } \\
\text { W36, W54, W55, W57, W69, } \\
\text { W70, W74, W77, W79, W85, } \\
\text { W86, W87, X00, X02, X04, } \\
\text { X06, X08, X09, X20, X30, } \\
\text { X32, X33, X44, X45, X48, } \\
\text { X49, X91, X94, X95, X99, } \\
\text { Y00, Y04, Y08, Y09, Y86, } \\
\text { Y88, Z88 }\end{array}$ & 84.9 & 96.0 \\
\hline Cancer & $\begin{array}{l}\text { C16, C14, C26, C22, C95, C55, } \\
\text { C34, C10, C71, C80, C15, C06, } \\
\text { C76, C32, C40, C41, C18, C39, } \\
\text { C64 }\end{array}$ & 61.1 & 96.0 \\
\hline Respiratory & $\mathrm{J} 45, \mathrm{~J} 18, \mathrm{~J} 44$ & 21.4 & 97.0 \\
\hline
\end{tabular}

\section{Cases}

In accordance with local coding conventions, I46 (cardiac arrest), R55 (syncope and collapse), and R96 (other sudden death, cause unknown) were included in the definition of ischemic heart disease. In practice, these adjustments made little absolute difference because the vast majority of premature mortality in this category were classified under I21 (88.9\%), I22 (3.9\%), and I25 $(3 \cdot 0 \%)$. 
Similarly, we included codes for hemiplegia and other paralytic syndromes (G81-G83) based on local coding conventions. In practice, this adjustment made little absolute difference because the vast majority of deaths in this category were classified under I64 (72.9\%), I69 (17.1\%), and I61 $(6 \cdot 5 \%)$.

\section{Controls}

After excluding deaths due to ischemic heart disease and stroke, we examined the remaining causes of death with at least 50 cases each. We eliminated any causes of death with a known etiological association with hypertension or diabetes based on a review of the literature. We first grouped together the causes of death related to injury. As injury deaths consisted predominantly of young men, we defined two additional control groups dying of cancer and respiratory conditions, which were the most common remaining causes of death. We pooled the control groups together for the main estimates, and generated additional estimates using each control group separately.

\section{High-Burden States}

There is a cluster of states in northeastern and eastern India that where the rate of stroke mortality is three times greater higher than the national average. ${ }^{2}$ We characterized these states as "high-burden" states. Because cardiovascular risk factor distributions might be different in these states, we analyzed this cluster separately for the stroke outcomes. The high-burden states are: Assam, West Bengal, Chhattisgarh, Sikkim, Arunachal Pradesh, Nagaland, Manipur, Mizoram, Tripura, and Meghalaya, for men and women; Odisha for women only.

\section{Time Trends}

We tested whether the effects of hypertension and diabetes varied by year. For ischemic heart disease, there was a significant interaction between hypertension and year $(\mathrm{p}=0.0002)$ and diabetes and year $(\mathrm{p}=0.0001)$. For stroke, there was a significant interaction between hypertension and year in both high- and low-burden states ( $\mathrm{p}=0.002$ and $\mathrm{p}=0.01$ respectively), and between diabetes and year in low-burden states only $(\mathrm{p}=0.002)$. These interaction terms were included in all logistic regression models. 


\section{Additional Results}

eTable 2 Baseline characteristics of case and control populations aged 30 to 69 years in the Million Death Study (2001 to 2014). All values in the table are unadjusted percentages unless otherwise indicated. Missing values were $<0.5 \%$ unless otherwise indicated.

\begin{tabular}{|c|c|c|c|c|c|c|c|}
\hline \multirow{3}{*}{ Characteristics } & \multicolumn{4}{|c|}{ Cases } & \multicolumn{3}{|c|}{ Controls } \\
\hline & \multirow{2}{*}{$\begin{array}{c}\text { Ischemic Heart } \\
\text { Disease } \\
(n=45,230)\end{array}$} & \multicolumn{3}{|c|}{ Stroke } & \multirow{2}{*}{$\begin{array}{c}\text { Injury } \\
(n=15,899)\end{array}$} & \multirow{2}{*}{$\begin{array}{c}\text { Cancer } \\
(n=22,197)\end{array}$} & \multirow{2}{*}{$\begin{array}{l}\text { Respiratory } \\
(n=22,353)\end{array}$} \\
\hline & & $\begin{array}{l}\text { High-Burden } \\
\quad(n=8,176)\end{array}$ & $\begin{array}{c}\text { Low-Burden } \\
(\mathrm{n}=14,275)\end{array}$ & $\begin{array}{c}\text { Total } \\
(\mathrm{n}=22,451)\end{array}$ & & & \\
\hline \multicolumn{8}{|l|}{ Previous Comorbidities } \\
\hline Diabetes & $6,056(13.4)$ & $967(11.8)$ & $1,826(12.8)$ & $2,793(12.4)$ & $273(1.7)$ & $958(4.3)$ & $875(3.9)$ \\
\hline Unknown & $3,299(7.3)$ & $947(11.6)$ & $1,004(7.0)$ & $1,951(8.7)$ & $1,290(8.1)$ & $1,798(8.1)$ & $1,834(8.2)$ \\
\hline Hypertension & $16,113(35.6)$ & $3,665(44.8)$ & $5,787(40.5)$ & $9,452(42.1)$ & $549(3.5)$ & $1,914(8.6)$ & $2,128(9.5)$ \\
\hline Unknown & $3,006(6.6)$ & $697(8.5)$ & $891(6.2)$ & $1,588(7.1)$ & $1,316(8.3)$ & $1,778(8.0)$ & $1,911(8.5)$ \\
\hline Heart Disease & $25,513(56.4)$ & $1,445(17.7)$ & $1,327(9.3)$ & $2,772(12.3)$ & $300(1.9)$ & $659(3.0)$ & $1,048(4.7)$ \\
\hline Unknown & $2,500(5.5)$ & $919(11.2)$ & $1,087(7.6)$ & $2,006(8.9)$ & $1,295(8.1)$ & $1,758(7.9)$ & $1,854(8.3)$ \\
\hline Stroke & $4,478(9.9)$ & $4,854(59.4)$ & $7,736(54.2)$ & $12,590(56.1)$ & $184(1.2)$ & $336(1.5)$ & $290(1.3)$ \\
\hline Unknown & $3,028(6.7)$ & $495(6.1)$ & $669(4.7)$ & $1,164(5.2)$ & $1,244(7.8)$ & $1,615(7.3)$ & $1,652(7.4)$ \\
\hline Cancer & $240(0.5)$ & $48(0.6)$ & $92(0.6)$ & $140(0.6)$ & $57(0.4)$ & $18,378(82.8)$ & $217(1.0)$ \\
\hline Unknown & $3,223(7.1)$ & $817(10.0)$ & $969(6.8)$ & $1,786(8.0)$ & $1,205(7.6)$ & $593(2.7)$ & $1,688(7.6)$ \\
\hline Asthma & $2,619(5.8)$ & $540(6.6)$ & $743(5.2)$ & $1,283(5.7)$ & $289(1.8)$ & $1,176(5.3)$ & $18,372(82.2)$ \\
\hline Unknown & $3,149(7.0)$ & $804(9.8)$ & $945(6.6)$ & $1,749(7.8)$ & $1,206(7.6)$ & $1,611(7.3)$ & $609(2.7)$ \\
\hline \multicolumn{8}{|c|}{ Medications (2004-14 only) } \\
\hline Taking medications & $8,819(23.2)$ & $1,339(19.6)$ & $3,881(34.5)$ & $5,220(28.9)$ & $672(5.1)$ & $7,193(39.5)$ & $5,923(34.3)$ \\
\hline Unknown & $6,562(17.3)$ & $1,404(20.5)$ & $1,659(14.8)$ & $3,063(16.9)$ & $1,997(15.1)$ & $2,918(16.0)$ & $2,989(17.3)$ \\
\hline Missing & $3,773(9.9)$ & $608(8.9)$ & $1,268(11.3)$ & $1,876(10.4)$ & $1,440(10.9)$ & $1,927(10.6)$ & $1,766(10.2)$ \\
\hline \multicolumn{8}{|l|}{ Smoking (men only) } \\
\hline None & $17,569(55.4)$ & $2,155(48.1)$ & $5,103(58.2)$ & $7,258(54.8)$ & $7,047(57.9)$ & $5,551(45.1)$ & $5,904(44.1)$ \\
\hline Cigarettes only & $2,436(7.7)$ & $334(7.5)$ & $379(4.3)$ & $713(5.4)$ & $752(6.2)$ & $996(8.1)$ & $474(3.5)$ \\
\hline Bidis only & $8,231(26.0)$ & $1,341(29.9)$ & $2,535(28.9)$ & $3,876(29.3)$ & $2,961(24.3)$ & $3,948(32.1)$ & $5,333(39.9)$ \\
\hline Cigarettes and bidis & $2,477(7.8)$ & $538(12.0)$ & $449(5.1)$ & $987(7.5)$ & $932(7.7)$ & $1,446(11.8)$ & $1,199(9.0)$ \\
\hline Missing & $999(3.2)$ & $114(2.5)$ & $296(3.4)$ & $410(3.1)$ & $486(4.0)$ & $360(2.9)$ & $465(3.5)$ \\
\hline \multicolumn{8}{|l|}{ Alcohol Use (men only) } \\
\hline None & $21,565(68.0)$ & $3,281(73.2)$ & $5,958(68.0)$ & $9,239(69.8)$ & $7,788(64.0)$ & $7,950(64.6)$ & $9,382(70.1)$ \\
\hline $1-4$ & $5,100(16.1)$ & $523(11.7)$ & $1,471(16.8)$ & $1,994(15.1)$ & $2,090(17.2)$ & $1,932(15.7)$ & $2,036(15.2)$ \\
\hline $5-7$ & $3,117(9.8)$ & $412(9.2)$ & $855(9.8)$ & $1,267(9.6)$ & $1,436(11.8)$ & $1,596(13.0)$ & $1,097(8.2)$ \\
\hline Unknown & $584(1.8)$ & $97(2.2)$ & $132(1.5)$ & $229(1.7)$ & $272(2.2)$ & $306(2.5)$ & $237(1.8)$ \\
\hline Missing & $1,346(4.2)$ & $169(3.8)$ & $346(3.9)$ & $515(3.9)$ & $592(4.9)$ & $517(4.2)$ & $623(4.7)$ \\
\hline
\end{tabular}


eFigure 1 (next page) Total and stratified estimates of the association between (A) hypertension or (B) diabetes and ischemic heart disease mortality, displayed separately for each control group. Estimates are adjusted for age, sex, rurality, region, smoking, alcohol use, and year. The area of each shape is proportional to the sample size (cases and controls). 


\section{A. Hypertension and Ischemic Heart Disease}

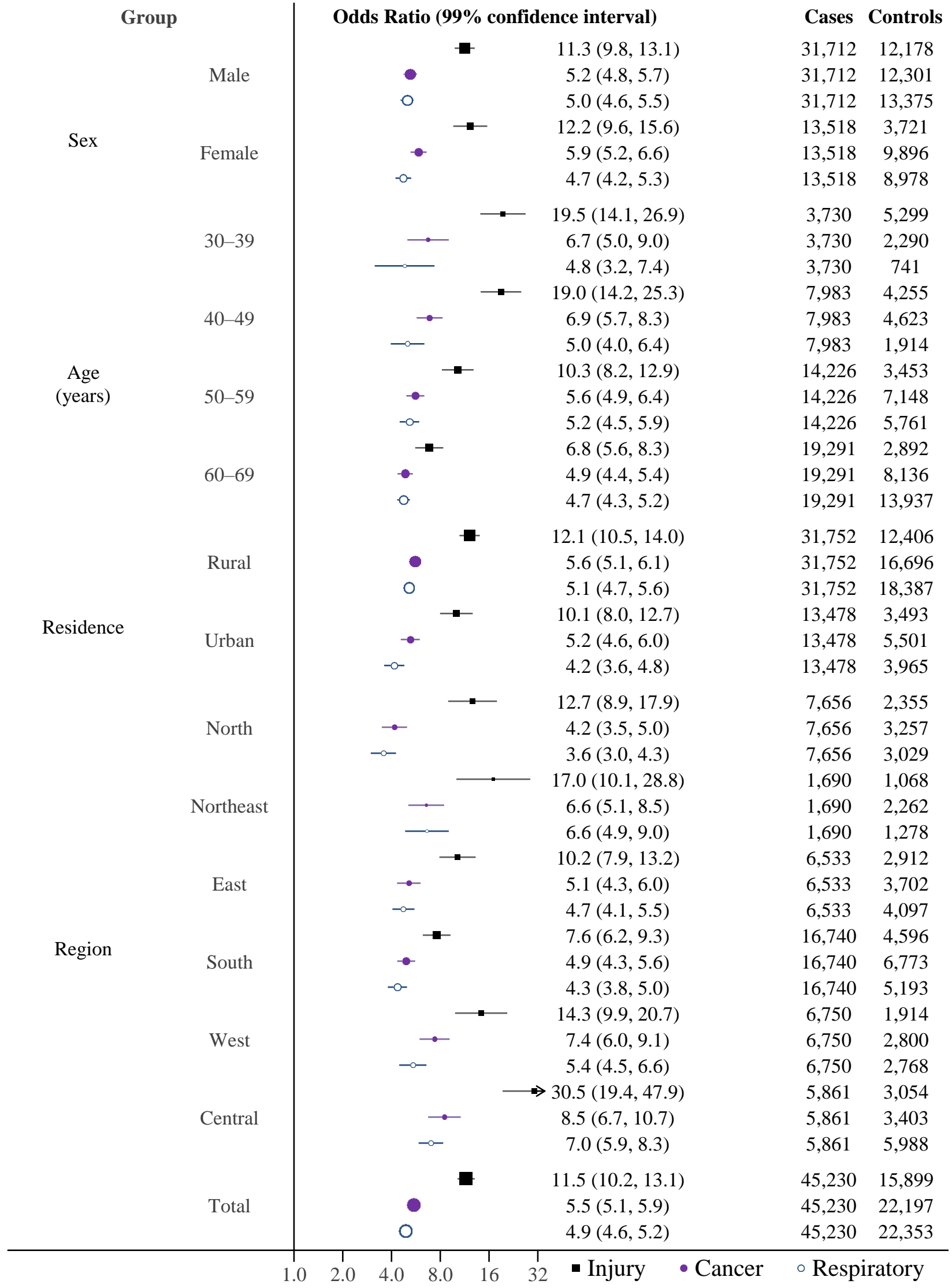




\section{B. Diabetes and Ischemic Heart Disease}

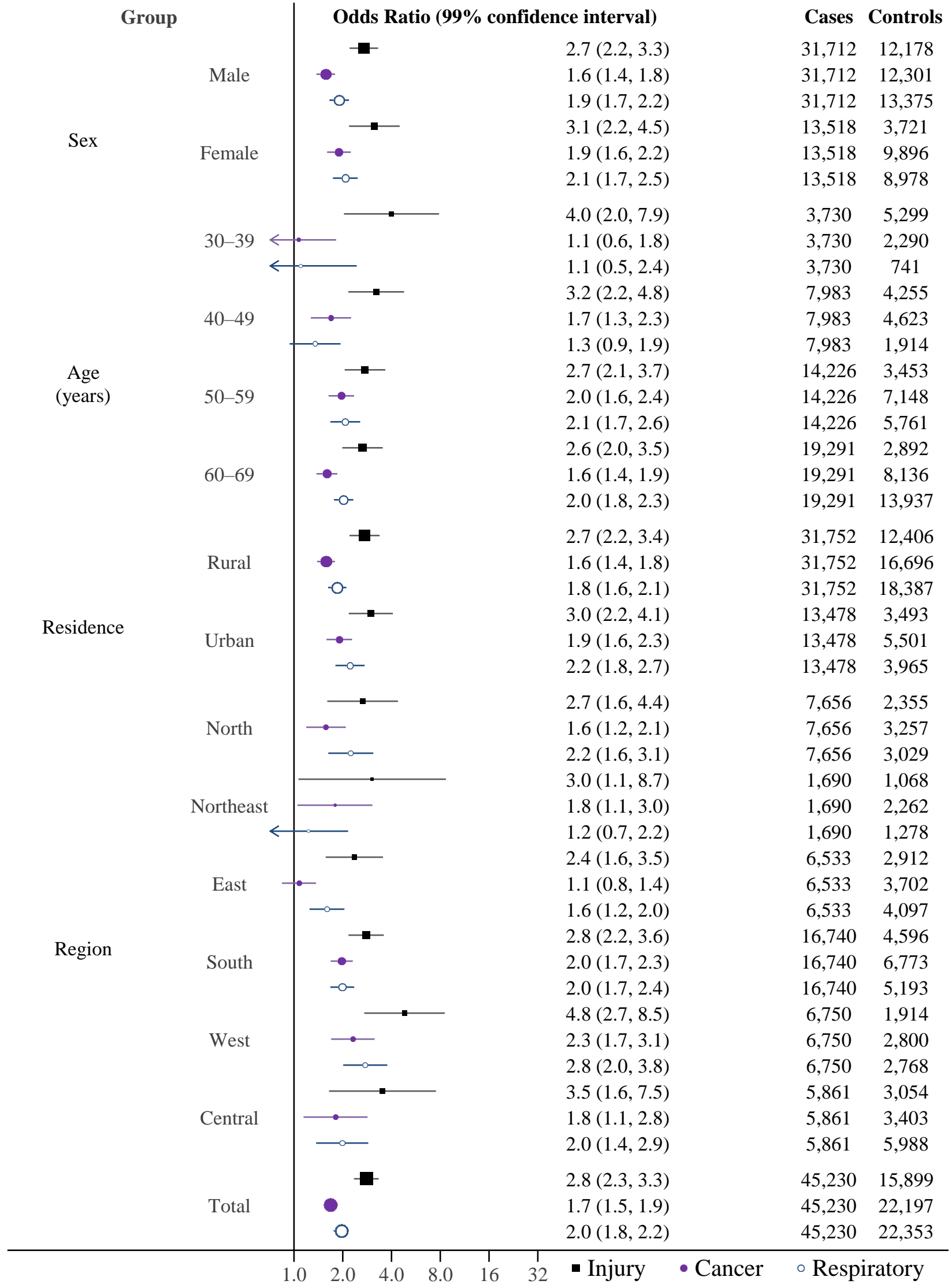


eFigure 2 Total and stratified estimates of the association between (A) hypertension or (B) diabetes and stroke mortality in high-burden states, displayed separately for each control group. Estimates are adjusted for age, sex, rurality, region, smoking, alcohol use, and year. The area of each shape is proportional to the sample size (cases and controls).

\section{A. Hypertension and Stroke, High-Burden States}

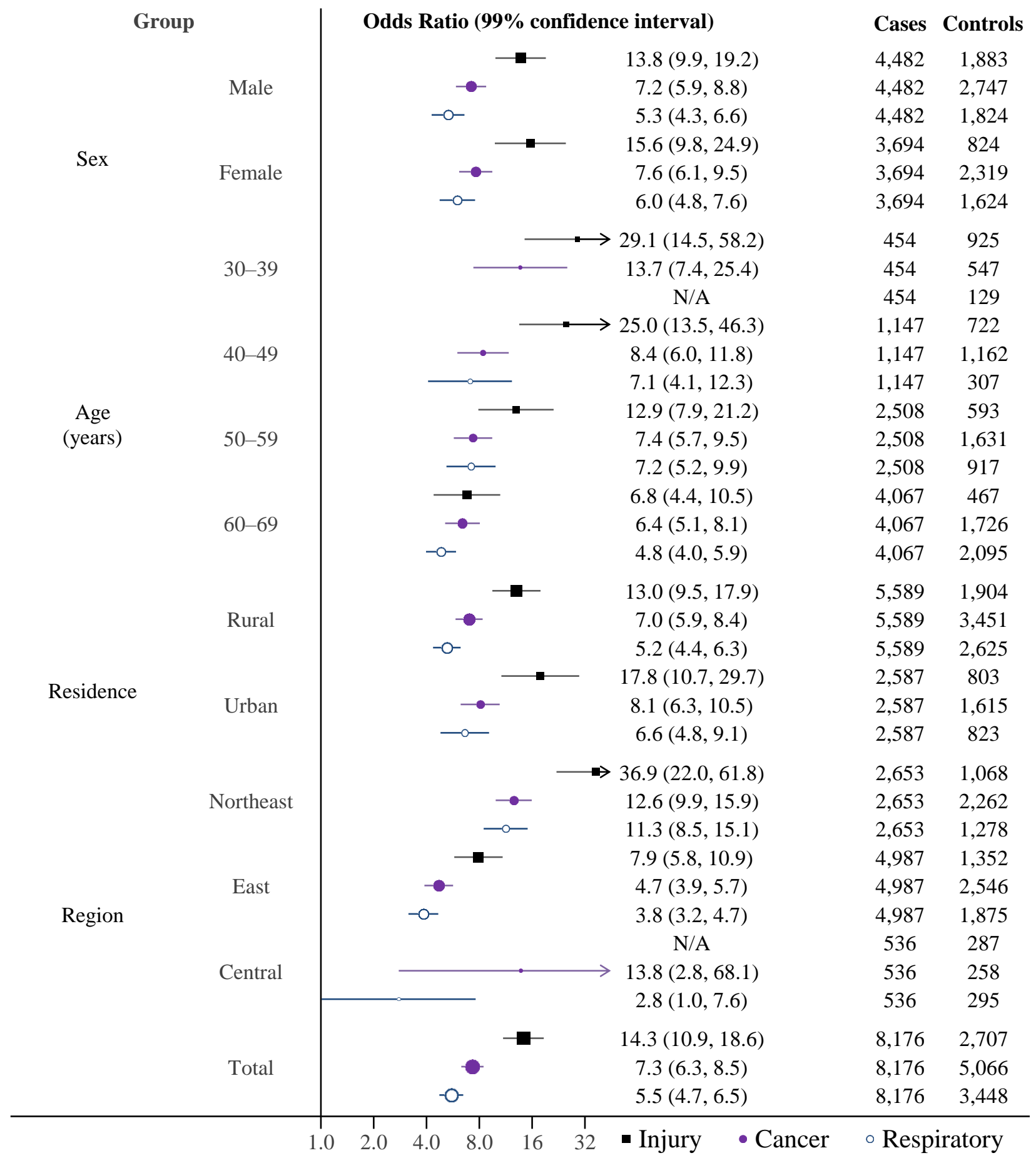




\section{B. Diabetes and Stroke, High-Burden States}

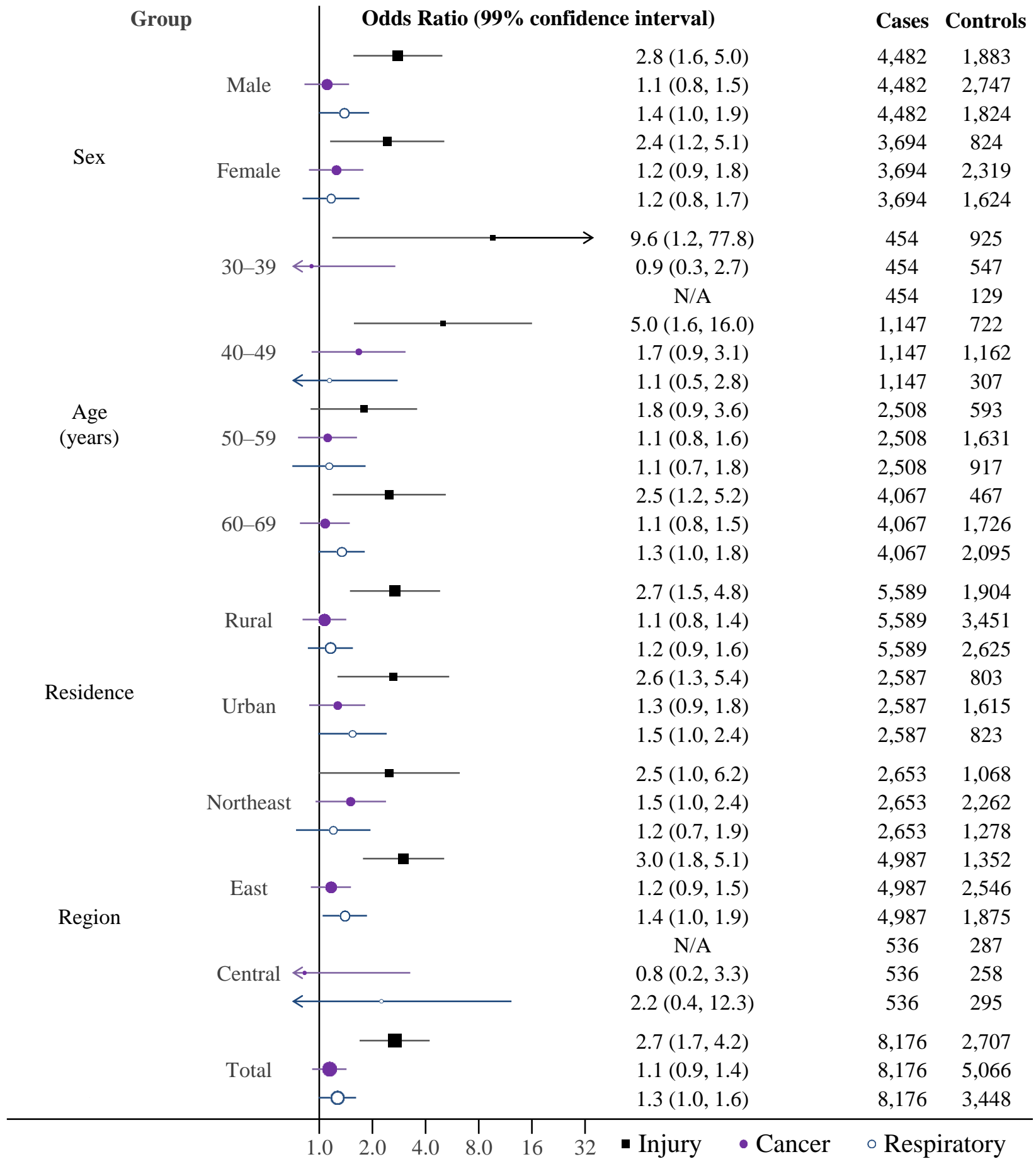


eFigure 3 (next page) Total and stratified estimates of the association between (A) hypertension or (B) diabetes and stroke mortality in low-burden states, displayed separately for each control group. Estimates are adjusted for age, sex, rurality, region, smoking, alcohol use, and year. The area of each shape is proportional to the sample size (cases and controls). 


\section{A. Hypertension and Stroke, Low-Burden States}

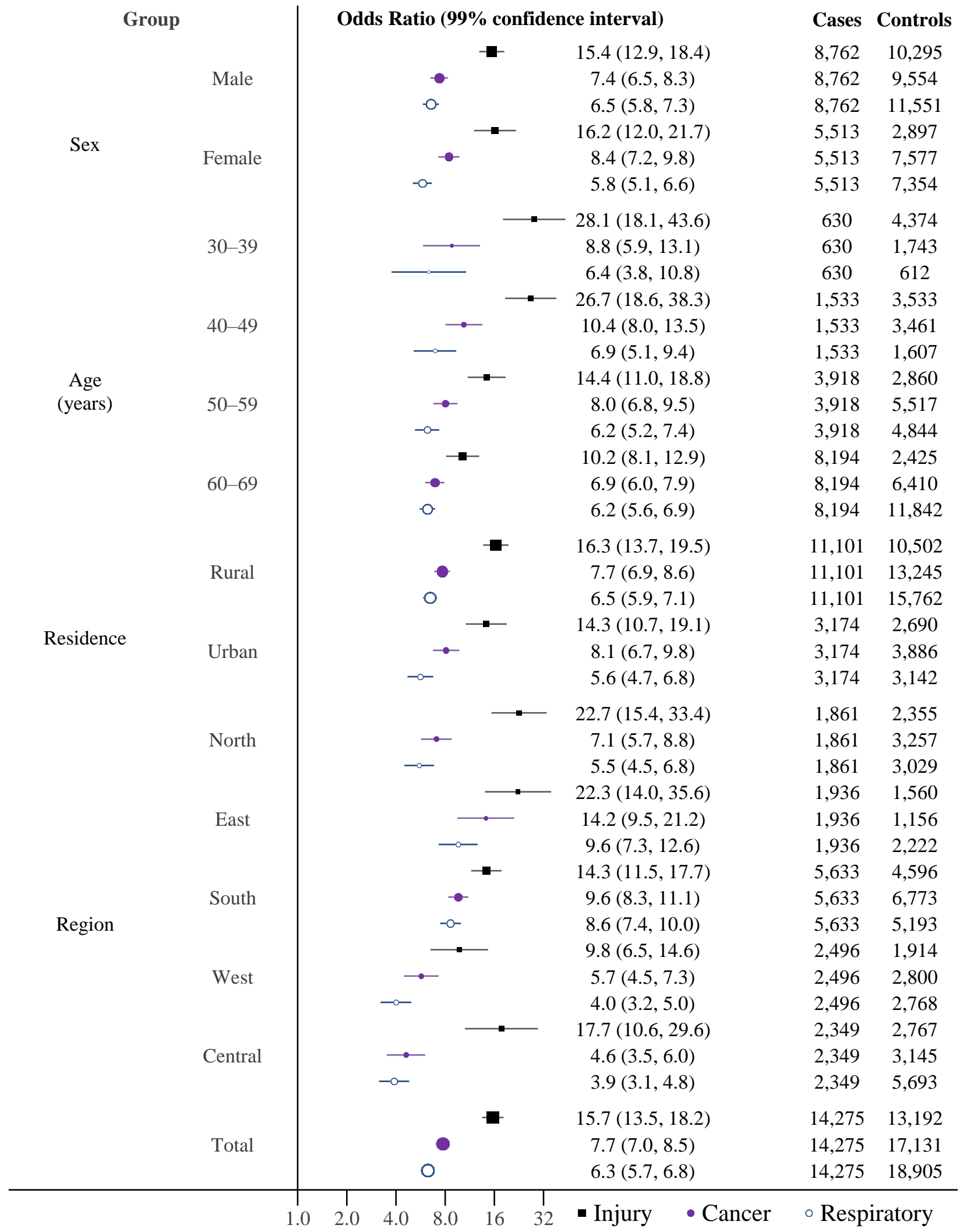




\section{B. Diabetes and Stroke, Low-Burden States}

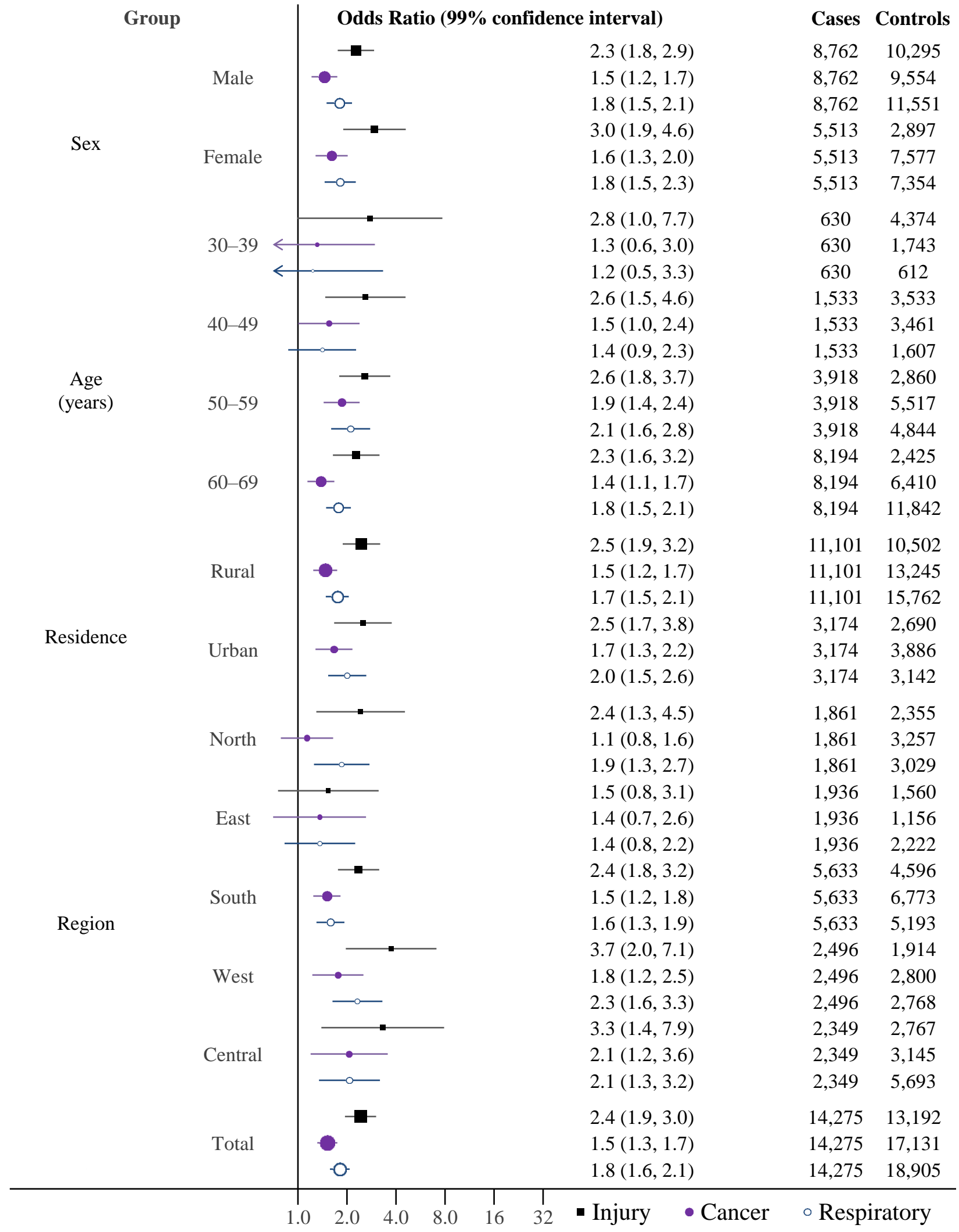


eFigure 4 (next page) Association between hypertension (A) or diabetes (B) and stroke mortality in high-burden states. The total and stratified estimates are adjusted for age, sex, urban/rural residence, region, smoking, alcohol use, and year. The area of each box is proportional to the sample size (cases and controls). 


\section{A. Hypertension and stroke, high-burden states}

\begin{tabular}{|c|c|c|c|c|}
\hline \multicolumn{2}{|c|}{ Group } & Odds Ratio (99\% confidence interval) & \multirow{3}{*}{$\begin{array}{l}\text { Cases } \\
4,482 \\
3,694\end{array}$} & \multirow{3}{*}{$\begin{array}{c}\text { Controls } \\
6,454 \\
4,767\end{array}$} \\
\hline S & Male & $7.1(6.1,8.2)$ & & \\
\hline Sex & Female & $7.3(6.2,8.7)$ & & \\
\hline \multirow{4}{*}{$\begin{array}{c}\text { Age } \\
\text { (years) }\end{array}$} & $30-39$ & $-17.0(10.6,27.4)$ & 454 & 1,601 \\
\hline & $40-49$ & $10.3(7.7,13.7)$ & 1,147 & 2,191 \\
\hline & $50-59$ & $7.7(6.3,9.5)$ & 2,508 & 3,141 \\
\hline & $60-69$ & $5.5(4.7,6.4)$ & 4,067 & 4,288 \\
\hline \multirow{2}{*}{ Residence } & Rural & $6.7(5.9,7.7)$ & 5,589 & 7,980 \\
\hline & Urban & $8.3(6.7,10.2)$ & 2,587 & 3,241 \\
\hline \multirow{3}{*}{ Region } & Northeast & $=\quad 14.0(11.6,17.0)$ & 2,653 & 4,608 \\
\hline & East & $4.7(4.1,5.4)$ & 4,987 & 5,773 \\
\hline & Central & $5.5(2.5,12.1)$ & 536 & 840 \\
\hline Total & & $7.1(6.4,8.0)$ & 8,176 & 11,221 \\
\hline
\end{tabular}

\section{B. Diabetes and stroke, high-burden states}

\begin{tabular}{|c|c|c|c|c|c|}
\hline \multicolumn{2}{|c|}{ Group } & \multicolumn{2}{|c|}{ Odds Ratio ( $99 \%$ confidence interval) } & \multirow{2}{*}{$\begin{array}{l}\text { Cases } \\
4,482\end{array}$} & \multirow{2}{*}{$\begin{array}{c}\text { Controls } \\
6,454\end{array}$} \\
\hline $\mathrm{S}_{0}$ & Male & 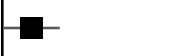 & $1.3(1.0,1.6)$ & & \\
\hline$\pi{ }^{2}$ & Female & 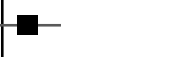 & $1.2(0.9,1.6)$ & 3,694 & 4,767 \\
\hline \multirow{4}{*}{$\begin{array}{c}\text { Age } \\
\text { (years) }\end{array}$} & $30-39$ & 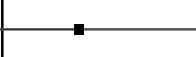 & $1.9(0.7,5.5)$ & 454 & 1,601 \\
\hline & $40-49$ & $\longrightarrow$ & $1.8(1.1,3.1)$ & 1,147 & 2,191 \\
\hline & $50-59$ & 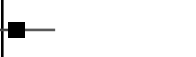 & $1.1(0.8,1.6)$ & 2,508 & 3,141 \\
\hline & $60-69$ & - & $1.2(1.0,1.6)$ & 4,067 & 4,288 \\
\hline \multirow{5}{*}{ Residence } & Rural & 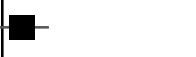 & $1.2(0.9,1.5)$ & 5,589 & 7,980 \\
\hline & Urban & - & $1.4(1.0,1.9)$ & 2,587 & 3,241 \\
\hline & Northeast & 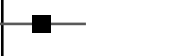 & $1.4(1.0,2.0)$ & 2,653 & 4,608 \\
\hline & East & - & $1.3(1.1,1.7)$ & 4,987 & 5,773 \\
\hline & Central & & $1.6(0.5,5.0)$ & 536 & 840 \\
\hline Total & & ta & $1.2(1.0,1.5)$ & 8,176 & 11,221 \\
\hline
\end{tabular}




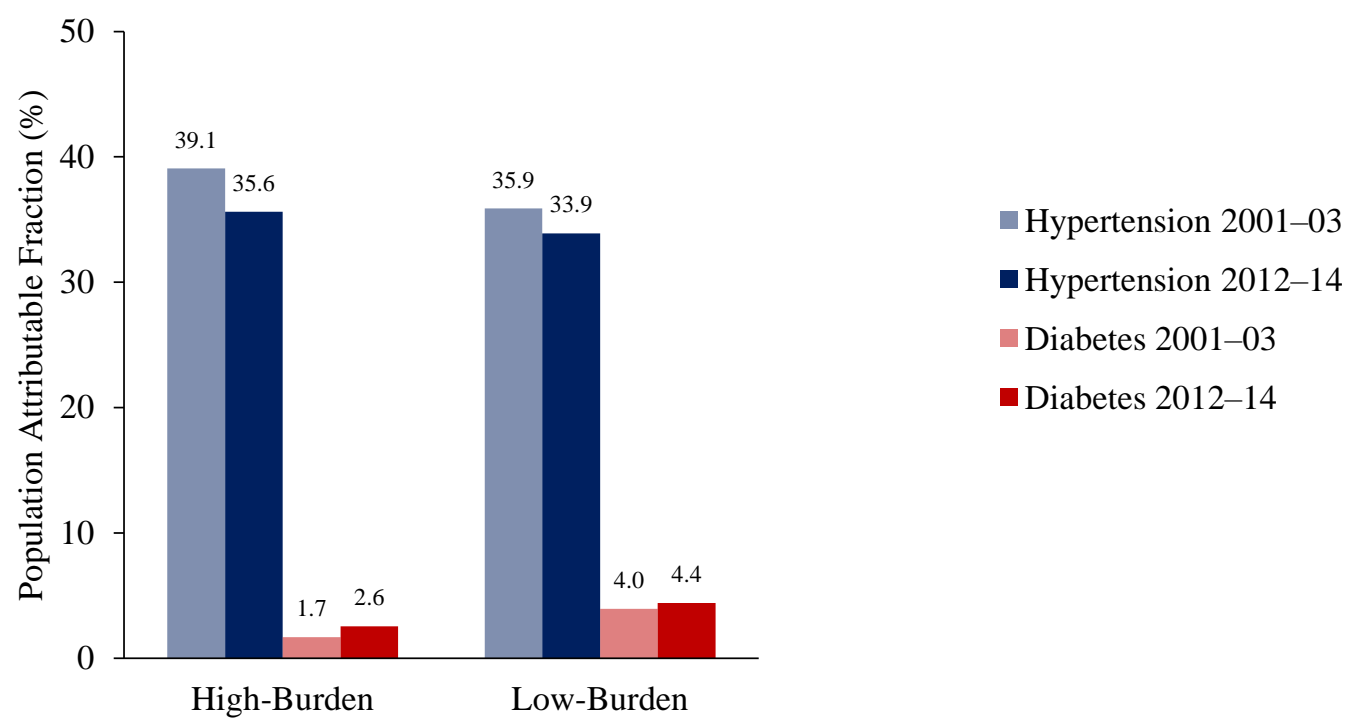

eFigure 5 Population attributable fractions (\%) for stroke in high- and low-burden states during the beginning (2001-03) and end (2012-14) of the study period. Blue bars represent hypertension; red bars represent diabetes. 
eFigure 6 (next page) Total and stratified estimates of the association between hypertension, diabetes, or both and (A) ischemic heart disease, (B) stroke (high-burden states), and (C) stroke (low-burden states). Estimates are adjusted for age, sex, rurality, region, smoking, alcohol use, and year. The area of each box is proportional to the sample size (cases and controls). 


\section{A. Ischemic Heart Disease}

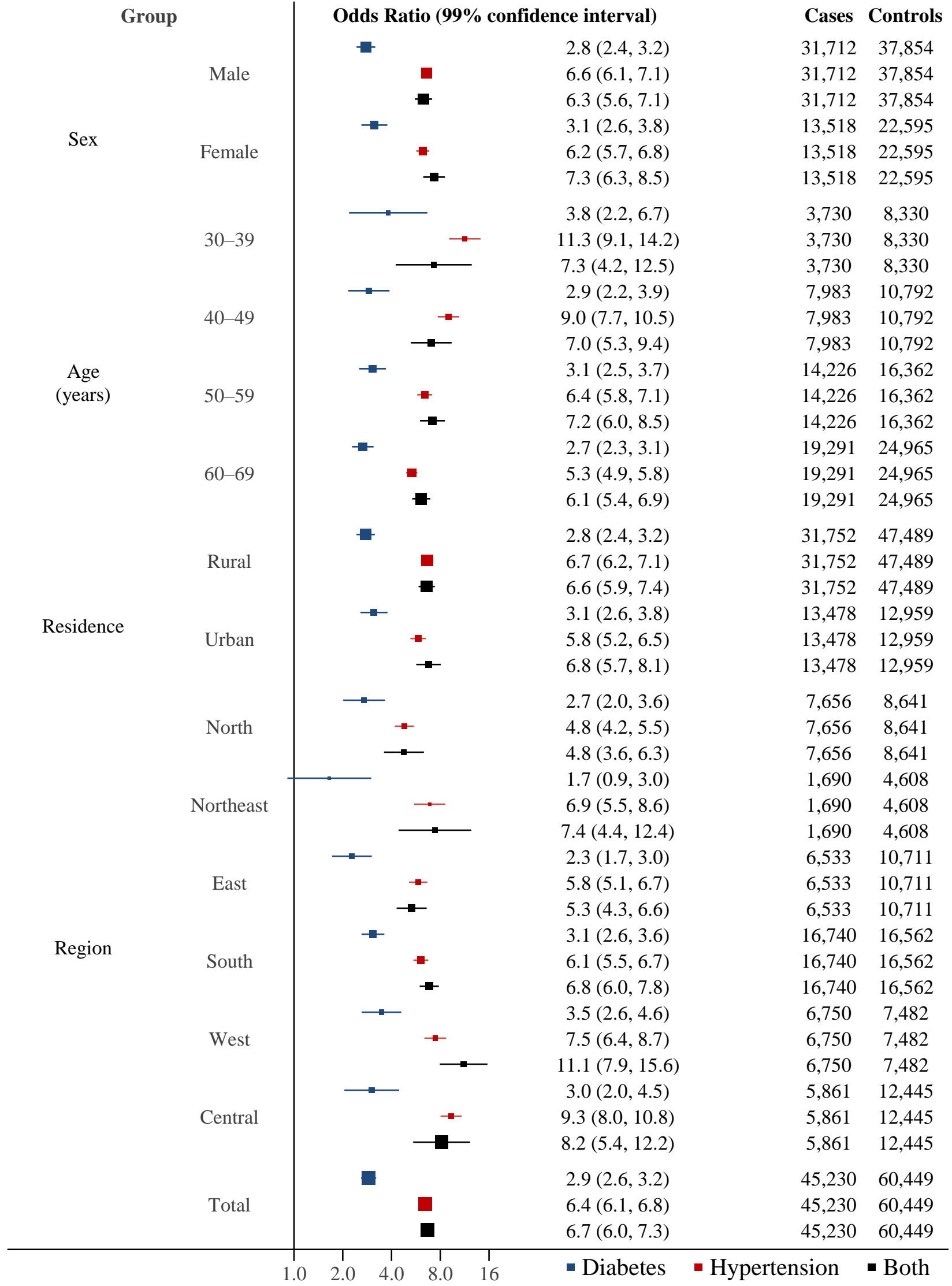




\section{B. Stroke, High-Burden States}

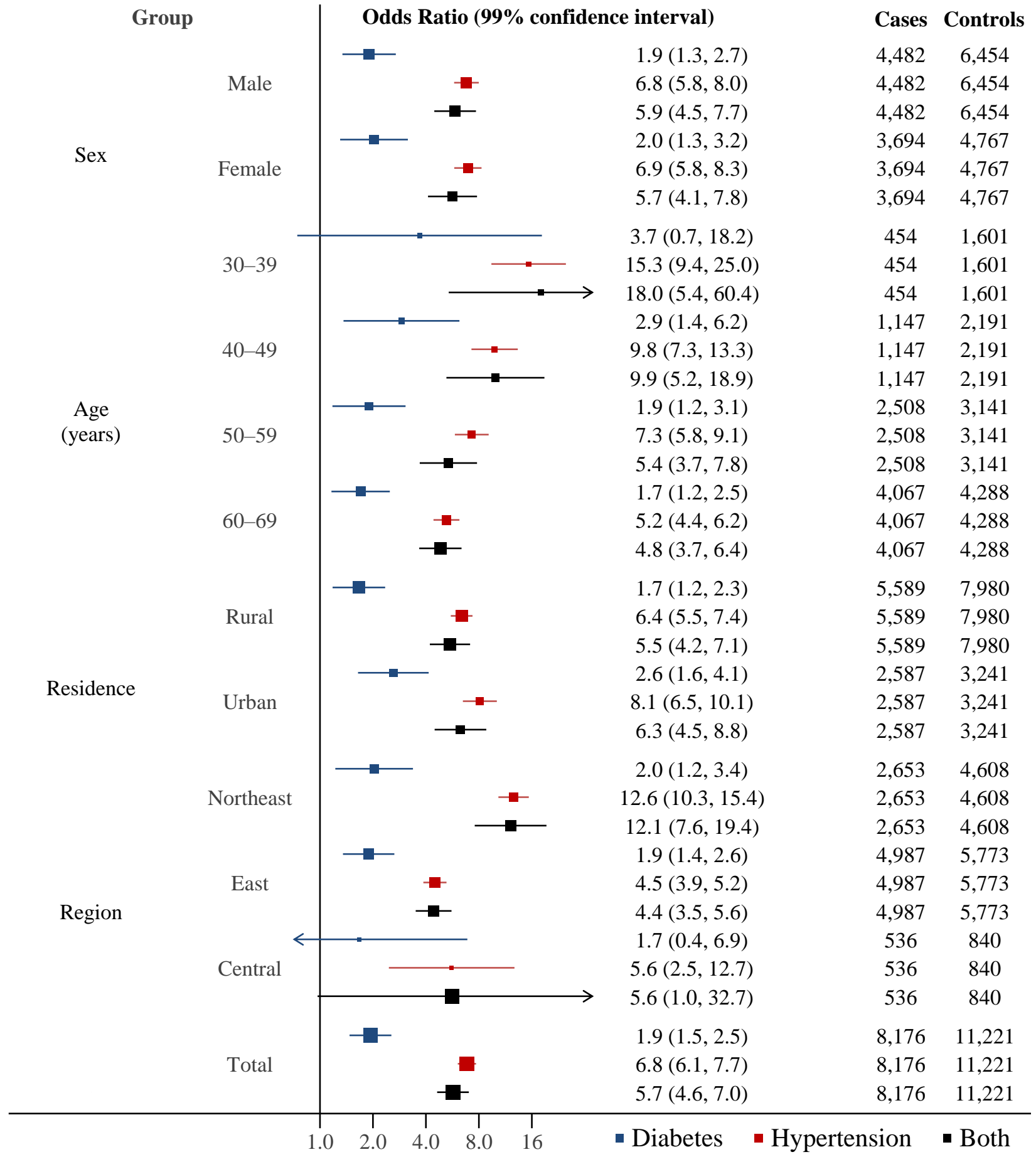




\section{Stroke, Low-Burden States}

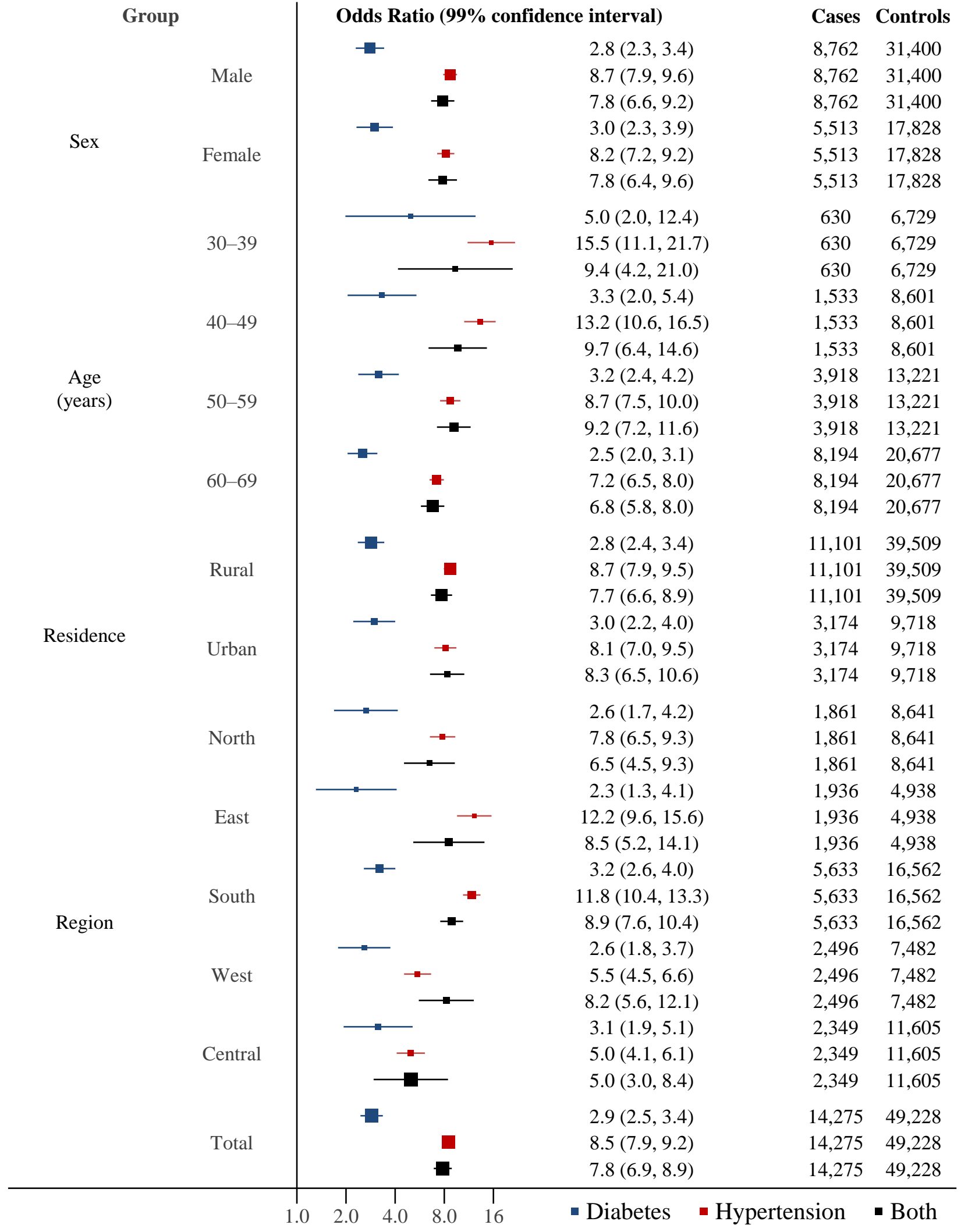


eTable 3 Association between hypertension and diabetes with ischemic heart disease and stroke by state. Some states located in the northeast region were combined due to low sample size. These states are s Sikkim, Arunachal Pradesh, Nagaland, Manipur, Mizoram, Tripura, and Meghalaya. High burden states for male stroke deaths were Assam, West Bengal, Chhattisgarh, and the northeast states. High burden states for female stroke deaths were Assam, West Bengal, Orissa, Chhattisgarh, and the northeastern states.

\begin{tabular}{|c|c|c|c|c|c|}
\hline Outcome & Risk Factor & State & Odds Ratio (99\% CI) & Cases & Controls \\
\hline \multirow{40}{*}{$\begin{array}{l}\text { Ischemic Heart } \\
\text { Disease }\end{array}$} & \multirow[t]{20}{*}{ Hypertension } & Bihar & $12.0(8.9-16.2)$ & 1801 & 2759 \\
\hline & & Uttar Pradesh & $11.9(9.5-14.8)$ & 2091 & 5531 \\
\hline & & Rajasthan & $9.8(7.6-12.6)$ & 1510 & 3428 \\
\hline & & Gujarat & $9.6(7.6-12.1)$ & 2657 & 3278 \\
\hline & & Orissa & $9.3(6.8-12.7)$ & 1046 & 2331 \\
\hline & & Madhya Pradesh & $9.2(6.2-13.7)$ & 1642 & 2645 \\
\hline & & Andhra Pradesh & $8.5(6.8-10.6)$ & 4107 & 3851 \\
\hline & & Chhattisgarh & $8.2(4.0-17.1)$ & 618 & 840 \\
\hline & & Northeast States & $7.6(5.8-10.0)$ & 1028 & 2827 \\
\hline & & Tamil Nadu & $7.6(6.2-9.3)$ & 4989 & 3891 \\
\hline & & Assam & $7.5(5.2-10.7)$ & 662 & 1781 \\
\hline & & Jharkhand & $6.9(4.3-11.1)$ & 613 & 782 \\
\hline & & Haryana & $6.5(4.3-9.9)$ & 1709 & 1895 \\
\hline & & Delhi & $5.9(3.6-9.5)$ & 926 & 824 \\
\hline & & Maharashtra & $5.5(4.4-6.9)$ & 2960 & 3159 \\
\hline & & Jammu \& Kashmir & $5.4(4.1-7.2)$ & 1104 & 1856 \\
\hline & & Karnataka & $3.9(3.2-4.8)$ & 3811 & 4617 \\
\hline & & West Bengal & $3.8(3.2-4.4)$ & 3073 & 4839 \\
\hline & & Kerala & $3.3(2.8-4.0)$ & 2939 & 3278 \\
\hline & & Punjab & $3.2(2.5-4.0)$ & 2458 & 1729 \\
\hline & \multirow{20}{*}{ Diabetes } & Kerala & $2.9(2.3-3.5)$ & 2939 & 3278 \\
\hline & & Tamil Nadu & $2.6(2.0-3.4)$ & 4989 & 3891 \\
\hline & & Rajasthan & $2.6(1.3-5.1)$ & 1510 & 3428 \\
\hline & & Maharashtra & $2.6(1.8-3.7)$ & 2960 & 3159 \\
\hline & & Chhattisgarh & $2.6(0.9-7.6)$ & 618 & 840 \\
\hline & & Haryana & $2.5(1.3-4.9)$ & 1709 & 1895 \\
\hline & & Madhya Pradesh & $2.4(1.1-5.3)$ & 1642 & 2645 \\
\hline & & Gujarat & $2.3(1.5-3.4)$ & 2657 & 3278 \\
\hline & & Delhi & $2.2(1.2-4.0)$ & 926 & 824 \\
\hline & & Orissa & $2.1(1.2-3.6)$ & 1046 & 2331 \\
\hline & & Karnataka & $1.9(1.4-2.5)$ & 3811 & 4617 \\
\hline & & Assam & $1.8(0.9-3.3)$ & 662 & 1781 \\
\hline & & Bihar & $1.6(1.0-2.7)$ & 1801 & 2759 \\
\hline & & Jammu \& Kashmir & $1.6(0.8-3.1)$ & 1104 & 1856 \\
\hline & & Uttar Pradesh & $1.5(0.9-2.3)$ & 2091 & 5531 \\
\hline & & Northeast States & $1.4(0.7-2.6)$ & 1028 & 2827 \\
\hline & & West Bengal & $1.4(1.1-1.7)$ & 3073 & 4839 \\
\hline & & Jharkhand & $1.3(0.6-3.1)$ & 613 & 782 \\
\hline & & Punjab & $1.2(0.9-1.8)$ & 2458 & 1729 \\
\hline & & Andhra Pradesh & $0.9(0.7-1.3)$ & 4107 & 3851 \\
\hline
\end{tabular}




\begin{tabular}{|c|c|c|c|c|c|}
\hline \multirow[t]{10}{*}{ Stroke, High Burden } & \multirow[t]{5}{*}{ Hypertension } & Assam & $23.6(17.3-32.2)$ & 1251 & 1781 \\
\hline & & $\begin{array}{l}\text { Northeast states (excluding } \\
\text { Assam) }\end{array}$ & $9.3(7.2-11.9)$ & 1402 & 2827 \\
\hline & & West Bengal & $4.1(3.5-4.8)$ & 4188 & 4839 \\
\hline & & Chhattisgarh & $5.5(2.5-12.1)$ & 536 & 840 \\
\hline & & Orissa (women) & & 799 & 934 \\
\hline & \multirow[t]{5}{*}{ Diabetes } & Chhattisgarh & $1.6(0.5-5.0)$ & 536 & 840 \\
\hline & & $\begin{array}{l}\text { Northeast states (excluding } \\
\text { Assam) }\end{array}$ & $1.5(0.9-2.5)$ & 1402 & 2827 \\
\hline & & West Bengal & $1.4(1.1-1.7)$ & 4188 & 4839 \\
\hline & & Assam & $1.2(0.7-2.0)$ & 1251 & 1781 \\
\hline & & Orissa (women) & & 799 & 934 \\
\hline \multirow[t]{32}{*}{ Stroke, Low Burden } & \multirow{16}{*}{ Hypertension } & Andhra Pradesh & $29.2(22.4-37.9)$ & 1445 & 3851 \\
\hline & & Kerala & $17.6(13.7-22.7)$ & 1087 & 3278 \\
\hline & & Bihar & $13.1(9.2-18.6)$ & 839 & 2759 \\
\hline & & Jammu \& Kashmir & $12.1(8.2-18.0)$ & 388 & 1856 \\
\hline & & Jharkhand & $11.0(6.2-19.6)$ & 254 & 782 \\
\hline & & Haryana & $9.7(5.7-16.4)$ & 359 & 1895 \\
\hline & & Punjab & $8.2(5.8-11.6)$ & 462 & 1729 \\
\hline & & Gujara & $2.6(2.2-3.0)$ & 737 & 3278 \\
\hline & & Rajasthan & $7.3(5.2-10.2)$ & 547 & 3428 \\
\hline & & Delhi & $5.6(2.8-11.3)$ & 226 & 824 \\
\hline & & Karnataka & $5.5(4.4-6.9)$ & 1560 & 4617 \\
\hline & & Tamil Nadu & $5.4(4.2-7.0)$ & 1193 & 3891 \\
\hline & & Uttar Pradesh & $5.3(4.0-7.2)$ & 887 & 5531 \\
\hline & & Madhya Pradesh & $4.8(3.0-7.7)$ & 915 & 2645 \\
\hline & & Maharashtra & $3.7(2.8-4.8)$ & 1326 & 3159 \\
\hline & & Orissa (men) & & 843 & 1397 \\
\hline & \multirow[t]{16}{*}{ Diabetes } & Madhya Pradesh & $3.6(1.6-8.0)$ & 915 & 2645 \\
\hline & & Tamil Nadu & $2.6(1.9-3.7)$ & 1193 & 3891 \\
\hline & & Haryana & $2.6(1.0-7.0)$ & 359 & 1895 \\
\hline & & Maharashtra & $2.3(1.5-3.6)$ & 1326 & 3159 \\
\hline & & Rajasthan & $1.7(0.7-4.2)$ & 547 & 3428 \\
\hline & & Karnataka & $1.7(1.2-2.3)$ & 1560 & 4617 \\
\hline & & Uttar Pradesh & $1.6(1.0-2.8)$ & 887 & 5531 \\
\hline & & Gujarat & $1.6(0.8-3.4)$ & 737 & 3278 \\
\hline & & Delhi & $1.5(0.6-3.6)$ & 226 & 824 \\
\hline & & Kerala & $1.3(0.9-1.7)$ & 1087 & 3278 \\
\hline & & Jharkhand & $1.2(0.4-3.5)$ & 254 & 782 \\
\hline & & Jammu \& Kashmir & $1.2(0.4-3.1)$ & 388 & 1856 \\
\hline & & Punjab & $1.1(0.7-1.9)$ & 462 & 1729 \\
\hline & & Andhra Pradesh & $0.8(0.5-1.2)$ & 1445 & 3851 \\
\hline & & Bihar & $0.7(0.4-1.5)$ & 839 & 2759 \\
\hline & & Orissa (men) & & 843 & 1397 \\
\hline
\end{tabular}

*Odds ratios for stroke in Orissa could not be computed due to sample size limitations.

Abbreviations: CI, confidence interval 
eFigure 7 (next page) Total and stratified estimates of the association between (A) hypertension or (B) diabetes and ischemic heart disease mortality, where both physicians initially agreed on cause of death category without further adjudication. Estimates are adjusted for age, sex, rurality, region, smoking, alcohol use, and year*. The area of each box is proportional to the sample size (cases and controls).

*We tested whether the effects of diabetes and hypertension varied by year. There was a significant interaction between diabetes and year $(\mathrm{p}=0.0001)$ and hypertension and year $(\mathrm{p}=0.0002)$. These interaction terms were included in all models. 


\section{A. Hypertension and Ischemic Heart Disease}

\begin{tabular}{|c|c|c|c|c|c|}
\hline \multicolumn{2}{|c|}{ Group } & \multicolumn{2}{|c|}{ Odds Ratio ( $99 \%$ confidence interval) } & \multirow{2}{*}{$\begin{array}{l}\text { Cases } \\
26,890\end{array}$} & \multirow{2}{*}{$\begin{array}{c}\text { Controls } \\
37,854\end{array}$} \\
\hline Soy & Male & - & $6.1(5.7,6.5)$ & & \\
\hline SeX & Female & - & $5.8(5.3,6.4)$ & 10,915 & 22,595 \\
\hline \multirow{4}{*}{$\begin{array}{c}\text { Age } \\
\text { (years) }\end{array}$} & $30-39$ & $\rightarrow$ & $10.2(8.2,12.8)$ & 3,098 & 8,330 \\
\hline & $40-49$ & - & $8.4(7.3,9.7)$ & 6,935 & 10,792 \\
\hline & $50-59$ & + & $5.9(5.4,6.6)$ & 12,154 & 16,362 \\
\hline & $60-69$ & $=$ & $5.1(4.7,5.5)$ & 15,618 & 24,965 \\
\hline \multirow{2}{*}{ Residence } & Rural & - & $6.3(5.9,6.7)$ & 26,397 & 47,489 \\
\hline & Urban & 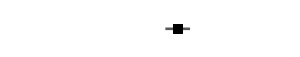 & $5.3(4.8,5.9)$ & 11,408 & 12,959 \\
\hline \multirow{6}{*}{ Region } & North & - & $4.5(4.0,5.2)$ & 6,588 & 8,641 \\
\hline & Northeast & $\rightarrow$ & $7.0(5.5,8.9)$ & 1,109 & 4,608 \\
\hline & East & - & $6.2(5.4,7.0)$ & 4,768 & 10,711 \\
\hline & South & - & $5.1(4.6,5.6)$ & 14,771 & 16,562 \\
\hline & West & $\rightarrow$ & $7.1(6.1,8.2)$ & 5,763 & 7,482 \\
\hline & Central & - & $10.0(8.6,11.6)$ & 4,806 & 12,445 \\
\hline Total & & घ & $6.0(5.7,6.3)$ & 37,805 & 60,449 \\
\hline
\end{tabular}

\section{B. Diabetes and Ischemic Heart Disease}

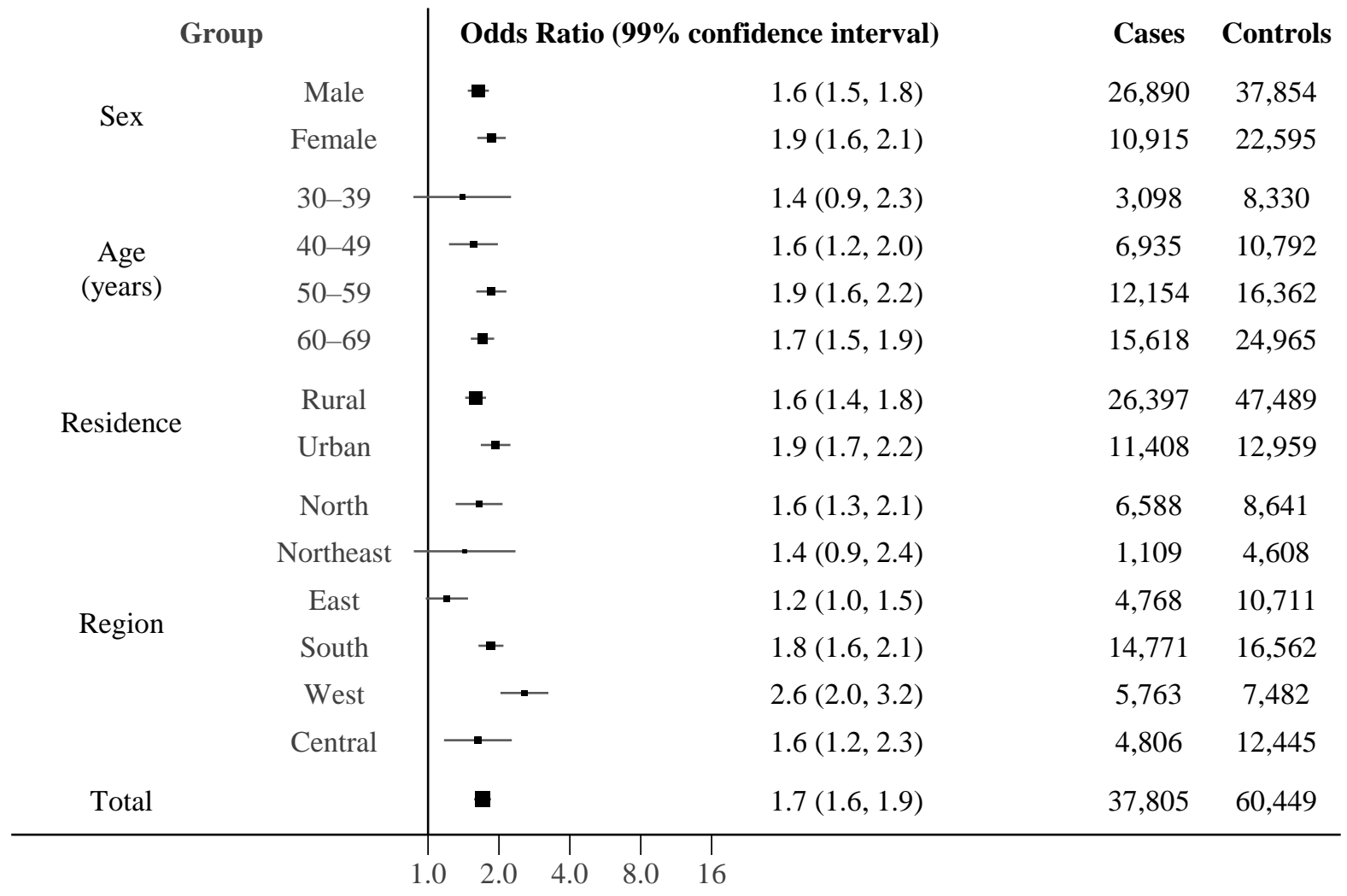


eFigure 8 Association between hypertension $(A, C)$ or diabetes $(B, D)$ and stroke mortality in high-burden (A,B) and low-burden (C,D) states, where both physicians initially agreed on cause of death category without further adjudication. The total and stratified estimates are adjusted for age, sex, urban/rural residence, region, smoking, alcohol use, and year*. The area of each box is proportional to the sample size (cases and controls).

*We tested whether the effects of diabetes and hypertension varied by year. There was a significant interaction between diabetes and year in low-burden states only $(p=0.002)$, and between hypertension and year in both high- and low-burden states $(\mathrm{p}=0.002$ and $\mathrm{p}=0.01$ respectively). These interaction terms were included in all models. 


\section{A. Hypertension and Stroke, High-Burden States}

\begin{tabular}{|c|c|c|c|c|c|}
\hline \multicolumn{2}{|c|}{ Group } & \multicolumn{2}{|c|}{ Odds Ratio ( $99 \%$ confidence interval) } & Cases & Controls \\
\hline \multirow{2}{*}{ Sex } & Male & $\rightarrow$ & $7.1(6.1,8.3)$ & 3,725 & 6,454 \\
\hline & Female & $\rightarrow$ & $7.2(6.1,8.6)$ & 3,095 & 4,767 \\
\hline \multirow{4}{*}{$\begin{array}{c}\text { Age } \\
\text { (years) }\end{array}$} & $30-39$ & $\longrightarrow$ & $-17.2(10.5,28.3)$ & 362 & 1,601 \\
\hline & $40-49$ & $\because$ & $11.0(8.2,15.0)$ & 953 & 2,191 \\
\hline & $50-59$ & $\rightarrow$ & $7.5(6.0,9.3)$ & 2,113 & 3,141 \\
\hline & $60-69$ & $\rightarrow$ & $5.4(4.6,6.4)$ & 3,392 & 4,288 \\
\hline \multirow{2}{*}{ Residence } & Rural & $=$ & $6.6(5.8,7.6)$ & 4,649 & 7,980 \\
\hline & Urban & $\rightarrow$ & $8.5(6.9,10.6)$ & 2,171 & 3,241 \\
\hline \multirow{3}{*}{ Region } & Northeast & $\rightarrow$ & $14.1(11.5,17.2)$ & 2,132 & 4,608 \\
\hline & East & - & $4.8(4.2,5.6)$ & 4,214 & 5,773 \\
\hline & Central & - & $5.3(2.3,12.2)$ & 474 & 840 \\
\hline Total & & $\because$ & $7.1(6.4,8.0)$ & 6,820 & 11,221 \\
\hline
\end{tabular}

\section{B. Diabetes and Stroke, High-Burden States}

\begin{tabular}{|c|c|c|c|c|c|}
\hline \multicolumn{2}{|c|}{ Group } & \multicolumn{2}{|c|}{ Odds Ratio (99\% confidence interval) } & \multirow{2}{*}{$\begin{array}{l}\text { Cases } \\
3,725\end{array}$} & \multirow{2}{*}{$\begin{array}{c}\text { Controls } \\
6,454\end{array}$} \\
\hline $\mathrm{C}_{3}$ & Male & - & $1.2(0.9,1.5)$ & & \\
\hline $\mathrm{Sex}$ & Female & - & $1.2(0.9,1.6)$ & 3,095 & 4,767 \\
\hline \multirow{4}{*}{$\begin{array}{c}\text { Age } \\
\text { (years) }\end{array}$} & $30-39$ & & $2.2(0.7,6.7)$ & 362 & 1,601 \\
\hline & $40-49$ & . & $1.6(0.9,2.9)$ & 953 & 2,191 \\
\hline & $50-59$ & - & $1.0(0.7,1.5)$ & 2,113 & 3,141 \\
\hline & $60-69$ & - & $1.2(0.9,1.5)$ & 3,392 & 4,288 \\
\hline \multirow{5}{*}{ Residence } & Rural & - & $1.1(0.9,1.4)$ & 4,649 & 7,980 \\
\hline & Urban & $=$ & $1.3(0.9,1.7)$ & 2,171 & 3,241 \\
\hline & Northeast & $=$ & $1.2(0.8,1.8)$ & 2,132 & 4,608 \\
\hline & East & $\rightarrow$ & $1.3(1.0,1.6)$ & 4,214 & 5,773 \\
\hline & Central & $\longrightarrow$ & $1.4(0.4,4.5)$ & 474 & 840 \\
\hline Total & & - & $1.2(1.0,1.4)$ & 6,820 & 11,221 \\
\hline
\end{tabular}




\section{Hypertension and Stroke, Low-Burden States}

\begin{tabular}{|c|c|c|c|c|c|}
\hline \multicolumn{2}{|c|}{ Group } & \multicolumn{2}{|c|}{ Odds Ratio ( $99 \%$ confidence interval) } & \multirow{2}{*}{$\begin{array}{l}\text { Cases } \\
6,996\end{array}$} & \multirow{2}{*}{$\begin{array}{c}\text { Controls } \\
31,400\end{array}$} \\
\hline Soy & Male & $=$ & $8.0(7.2,8.8)$ & & \\
\hline SeX & Female & 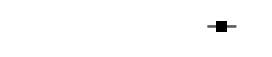 & $7.5(6.7,8.5)$ & 4,395 & 17,828 \\
\hline \multirow{4}{*}{$\begin{array}{c}\text { Age } \\
\text { (years) }\end{array}$} & $30-39$ & & $=-16.3(11.4,23.4)$ & 460 & 6,729 \\
\hline & $40-49$ & & $12.5(9.9,15.8)$ & 1,160 & 8,601 \\
\hline & $50-59$ & $\rightarrow$ & $8.0(6.9,9.2)$ & 3,135 & 13,221 \\
\hline & $60-69$ & 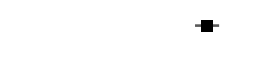 & $6.7(6.0,7.4)$ & 6,636 & 20,677 \\
\hline \multirow{2}{*}{ Residence } & Rural & $=$ & $7.9(7.3,8.7)$ & 8,884 & 39,509 \\
\hline & Urban & - & $7.6(6.5,8.8)$ & 2,507 & 9,718 \\
\hline \multirow{5}{*}{ Region } & North & $\rightarrow$ & $7.4(6.1,9.0)$ & 1,398 & 8,641 \\
\hline & East & & $-\quad 12.9(10.1,16.5)$ & 1,450 & 4,938 \\
\hline & South & - & $10.3(9.1,11.6)$ & 4,561 & 16,562 \\
\hline & West & $\rightarrow$ & $5.1(4.2,6.1)$ & 2,019 & 7,482 \\
\hline & Central & $\rightarrow$ & $4.6(3.7,5.6)$ & 1,963 & 11,605 \\
\hline Total & & घ & $7.8(7.2,8.4)$ & 11,391 & 49,228 \\
\hline
\end{tabular}

\section{Diabetes and Stroke, Low-Burden States}

\begin{tabular}{|c|c|c|c|c|c|}
\hline \multicolumn{2}{|c|}{ Group } & \multicolumn{2}{|c|}{ Odds Ratio ( $99 \%$ confidence interval) } & \multirow{2}{*}{$\begin{array}{l}\text { Cases } \\
6,996\end{array}$} & \multirow{2}{*}{$\begin{array}{c}\text { Controls } \\
31,400\end{array}$} \\
\hline $\mathrm{Cor}$ & Male & $\rightarrow$ & $1.3(1.1,1.5)$ & & \\
\hline Sex & Female & $\rightarrow$ & $1.4(1.1,1.7)$ & 4,395 & 17,828 \\
\hline \multirow{4}{*}{$\begin{array}{c}\text { Age } \\
\text { (years) }\end{array}$} & $30-39$ & & $0.9(0.4,2.2)$ & 460 & 6,729 \\
\hline & $40-49$ & - & $1.1(0.7,1.7)$ & 1,160 & 8,601 \\
\hline & $50-59$ & $\rightarrow$ & $1.5(1.2,1.9)$ & 3,135 & 13,221 \\
\hline & $60-69$ & - & $1.3(1.1,1.5)$ & 6,636 & 20,677 \\
\hline \multirow{2}{*}{ Residence } & Rural & 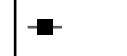 & $1.3(1.1,1.5)$ & 8,884 & 39,509 \\
\hline & Urban & $\rightarrow$ & $1.5(1.2,1.8)$ & 2,507 & 9,718 \\
\hline \multirow{5}{*}{ Region } & North & - & $1.1(0.7,1.5)$ & 1,398 & 8,641 \\
\hline & East & - & $1.0(0.6,1.6)$ & 1,450 & 4,938 \\
\hline & South & - & $1.2(1.0,1.4)$ & 4,561 & 16,562 \\
\hline & West & $\because$ & $1.8(1.4,2.5)$ & 2,019 & 7,482 \\
\hline & Central & $\longrightarrow$ & $1.6(1.1,2.5)$ & 1,963 & 11,605 \\
\hline Total & & 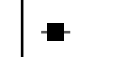 & $1.3(1.2,1.5)$ & 11,391 & 49,228 \\
\hline
\end{tabular}


eFigure 9 Total and stratified estimates of the association between (A) hypertension or (B) diabetes and ischemic heart disease mortality, displayed separately for men and women. Estimates are adjusted for age, rurality, region, smoking, alcohol use, and year. The area of each box is proportional to the sample size (cases and controls).

\section{A. Hypertension and Ischemic Heart Disease}

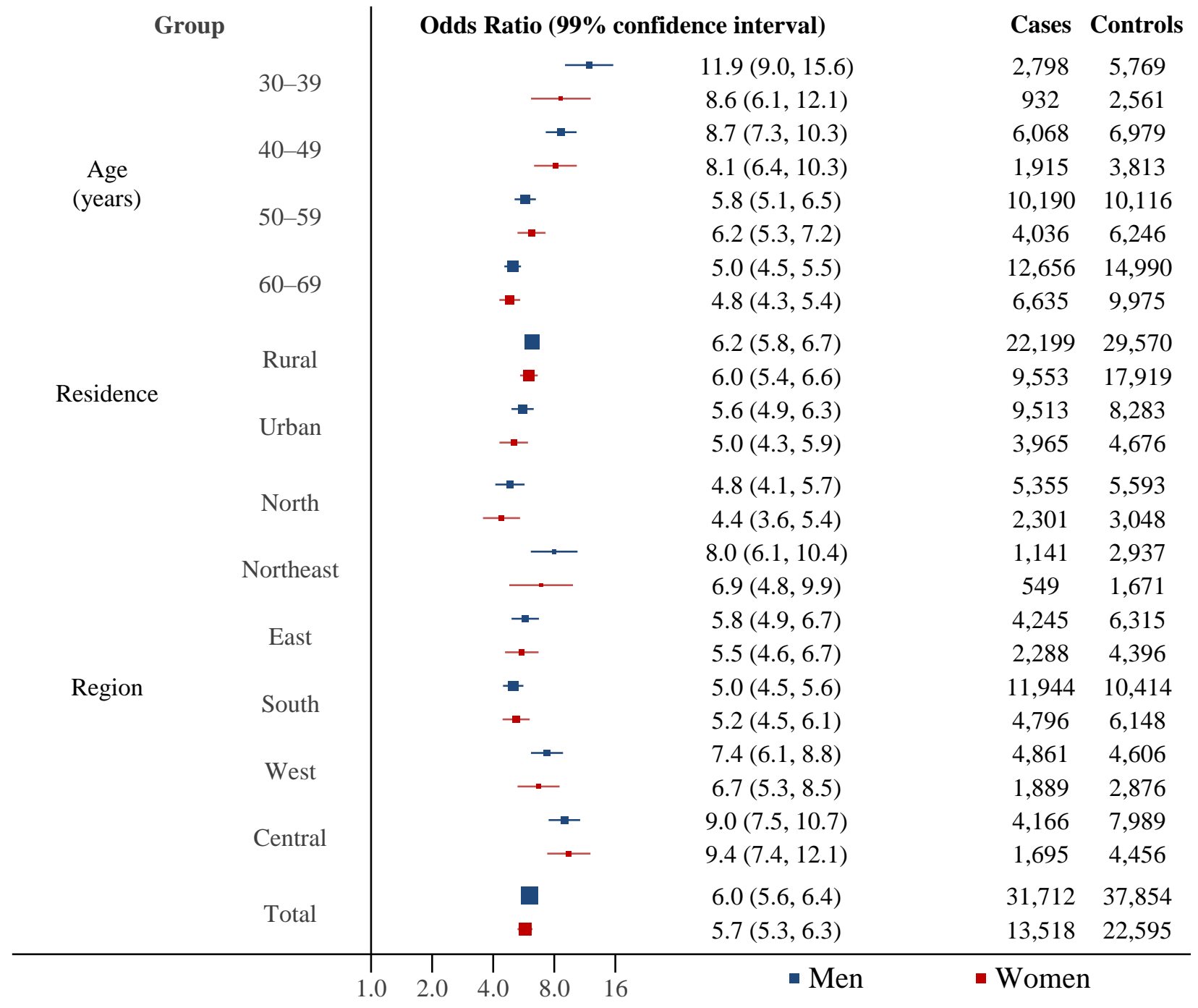




\section{B. Diabetes and Ischemic Heart Disease}

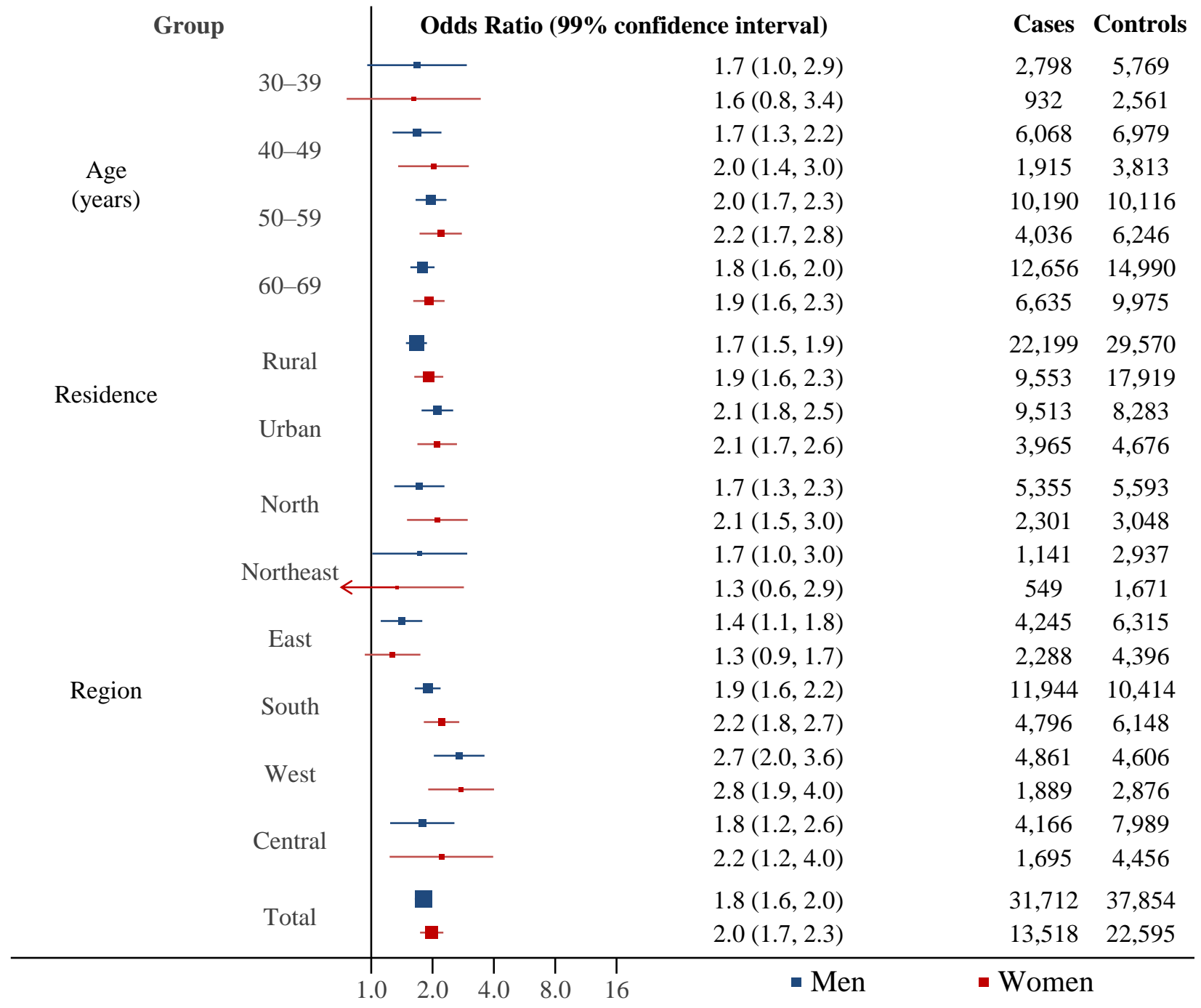


eFigure 10 Total and stratified estimates of the association between (A) hypertension or (B) diabetes and stroke mortality in high-burden states, displayed separately for men and women. Estimates are adjusted for age, rurality, region, smoking, alcohol use, and year. The area of each box is proportional to the sample size (cases and controls).

\section{A. Hypertension and Stroke, High-Burden States}

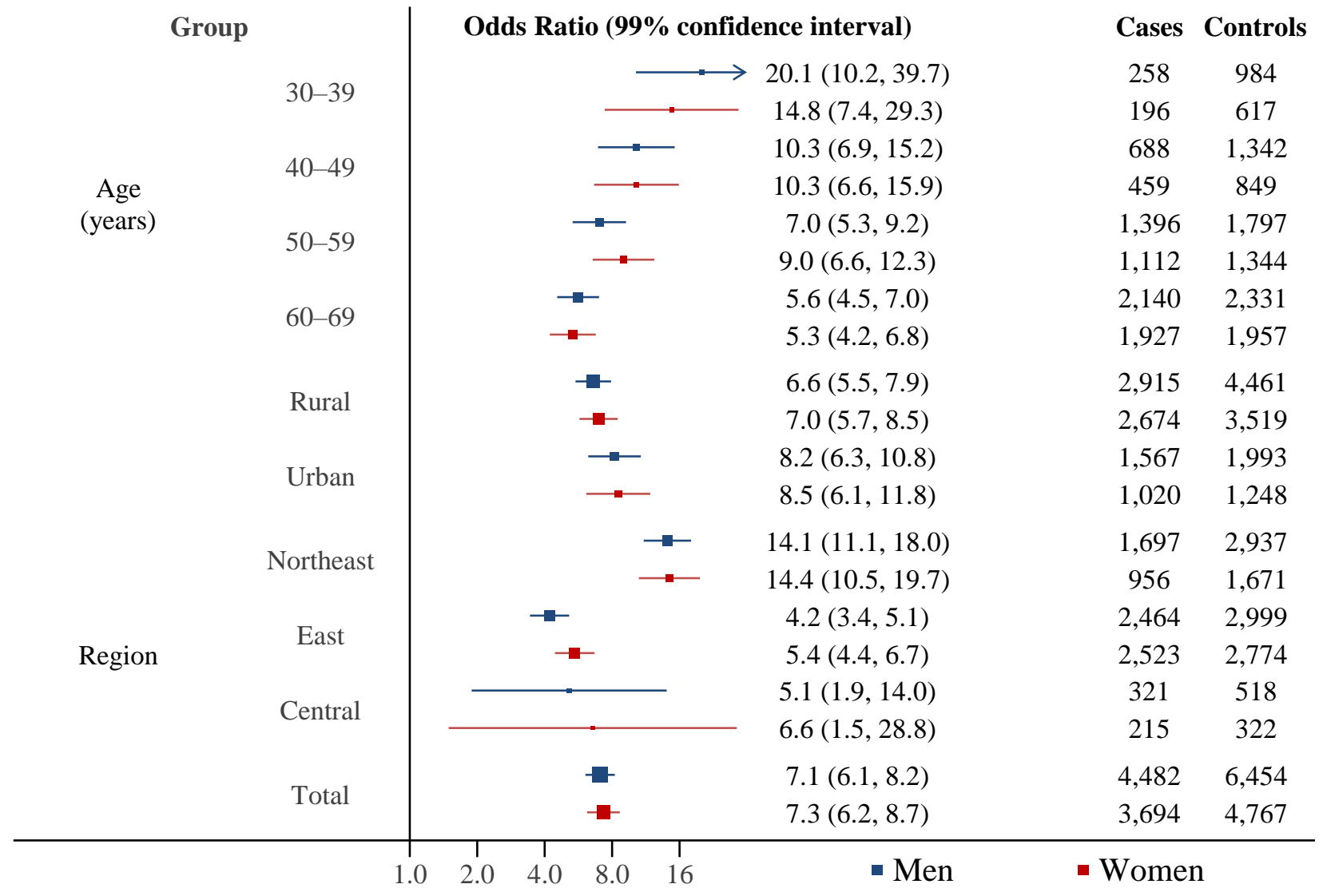




\section{B. Diabetes and Stroke, High-Burden States}

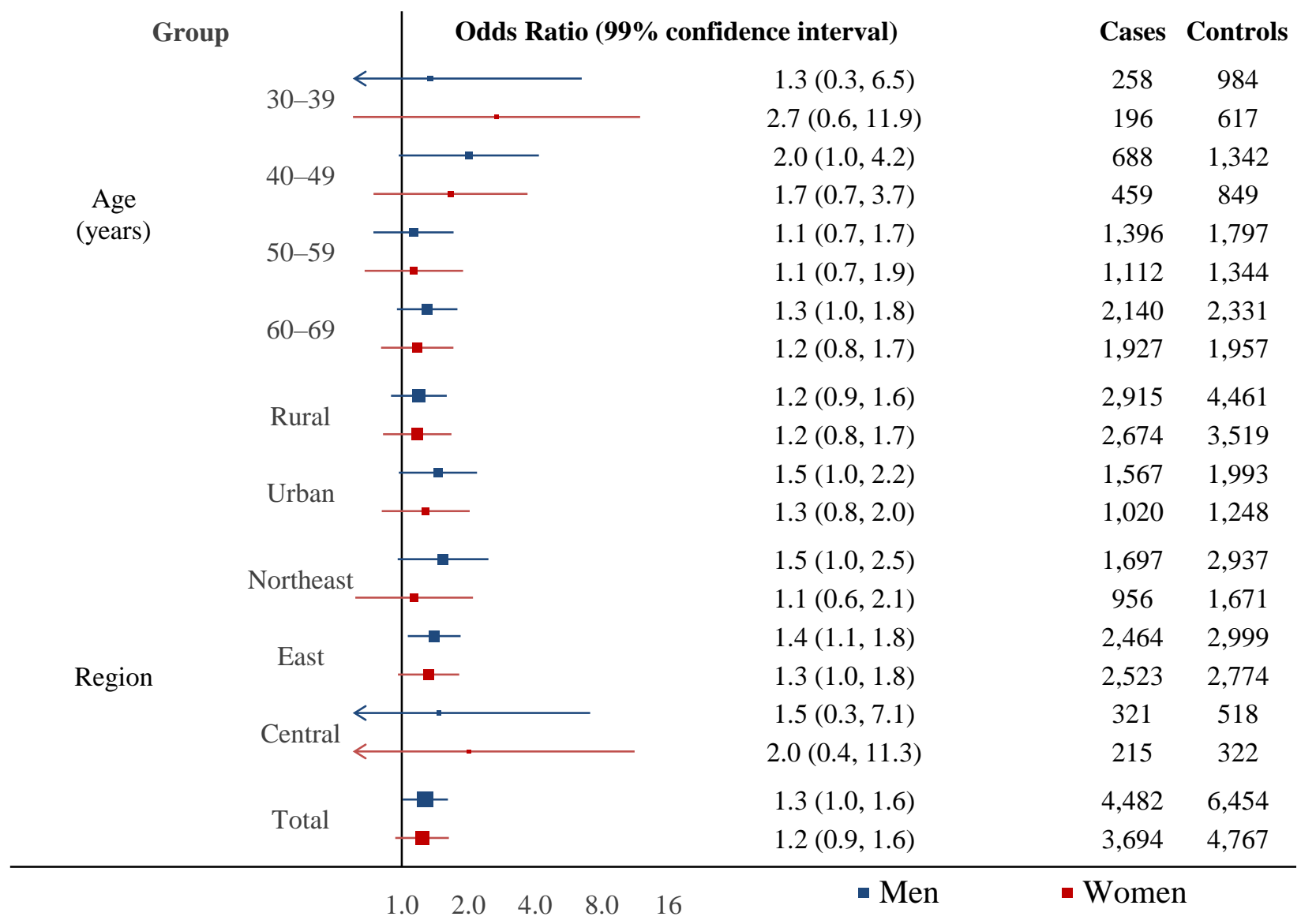


eFigure 11 Total and stratified estimates of the association between (A) hypertension or (B) diabetes and stroke mortality in low-burden states, displayed separately for men and women. Estimates are adjusted for age, rurality, region, smoking, alcohol use, and year. The area of each box is proportional to the sample size (cases and controls).

Abbreviation: N/A, cannot be estimated due to limited data

\section{A. Hypertension and Stroke, Low-Burden}

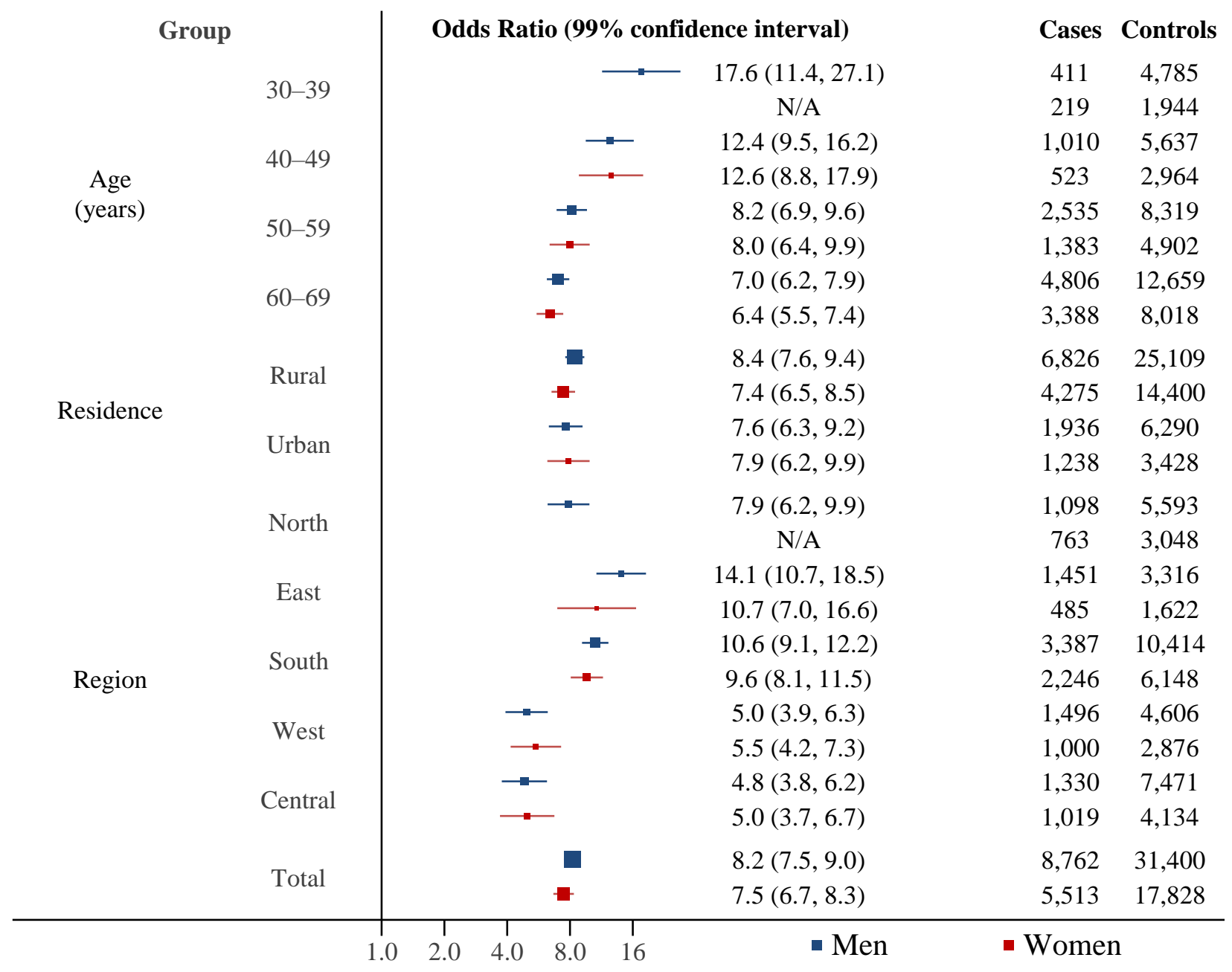




\section{B. Diabetes and Stroke, Low-Burden}

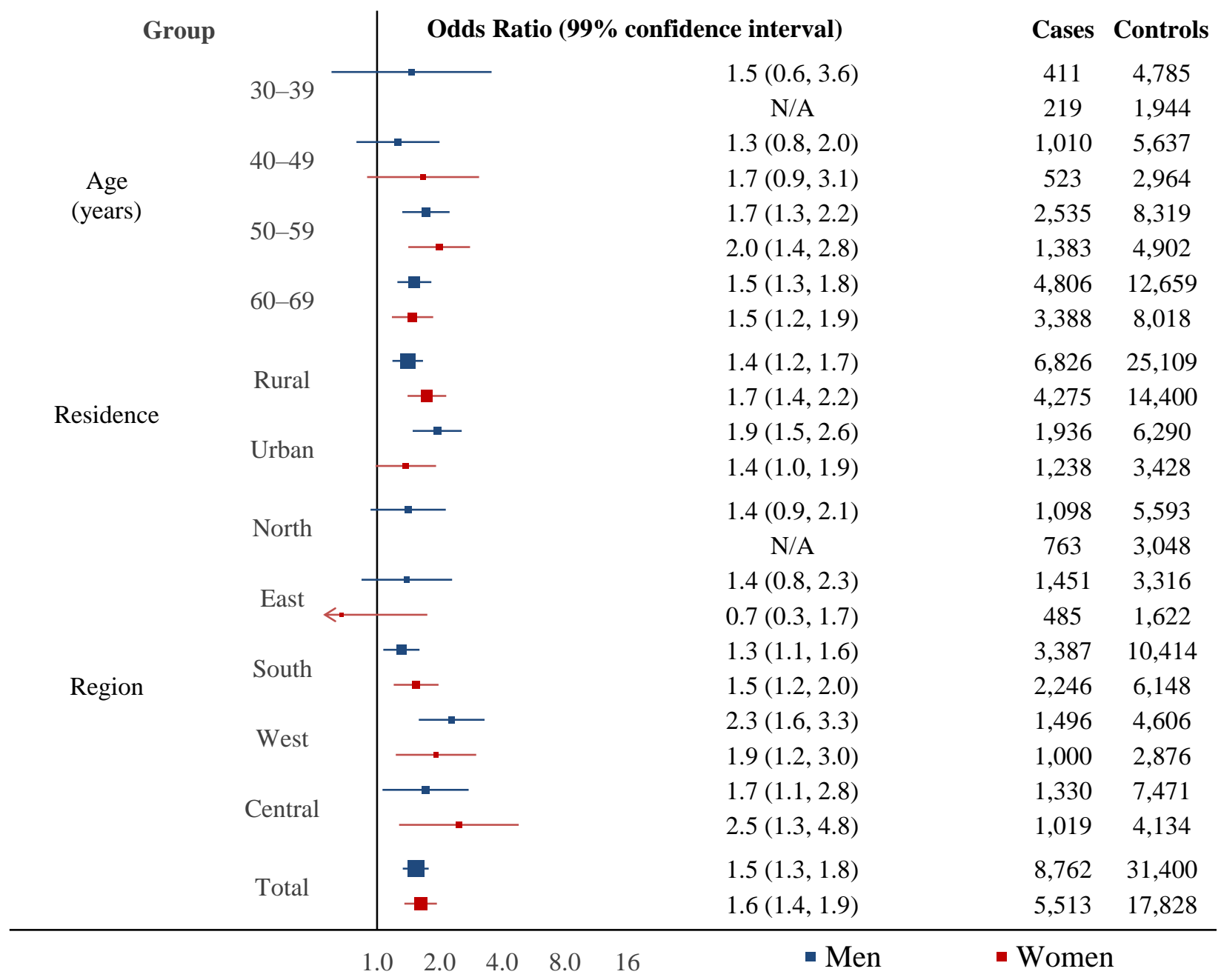


eFigure 12 Total and stratified estimates of the association between (A) hypertension or (B) diabetes and ischemic heart disease mortality, with and without socioeconomic status (SES) adjustment (2001 to 2013 only). Estimates are adjusted for age, sex, rurality, region, smoking, alcohol use, and year. The area of each box is proportional to the sample size (cases and controls).

\section{A. Hypertension and Ischemic Heart Disease}

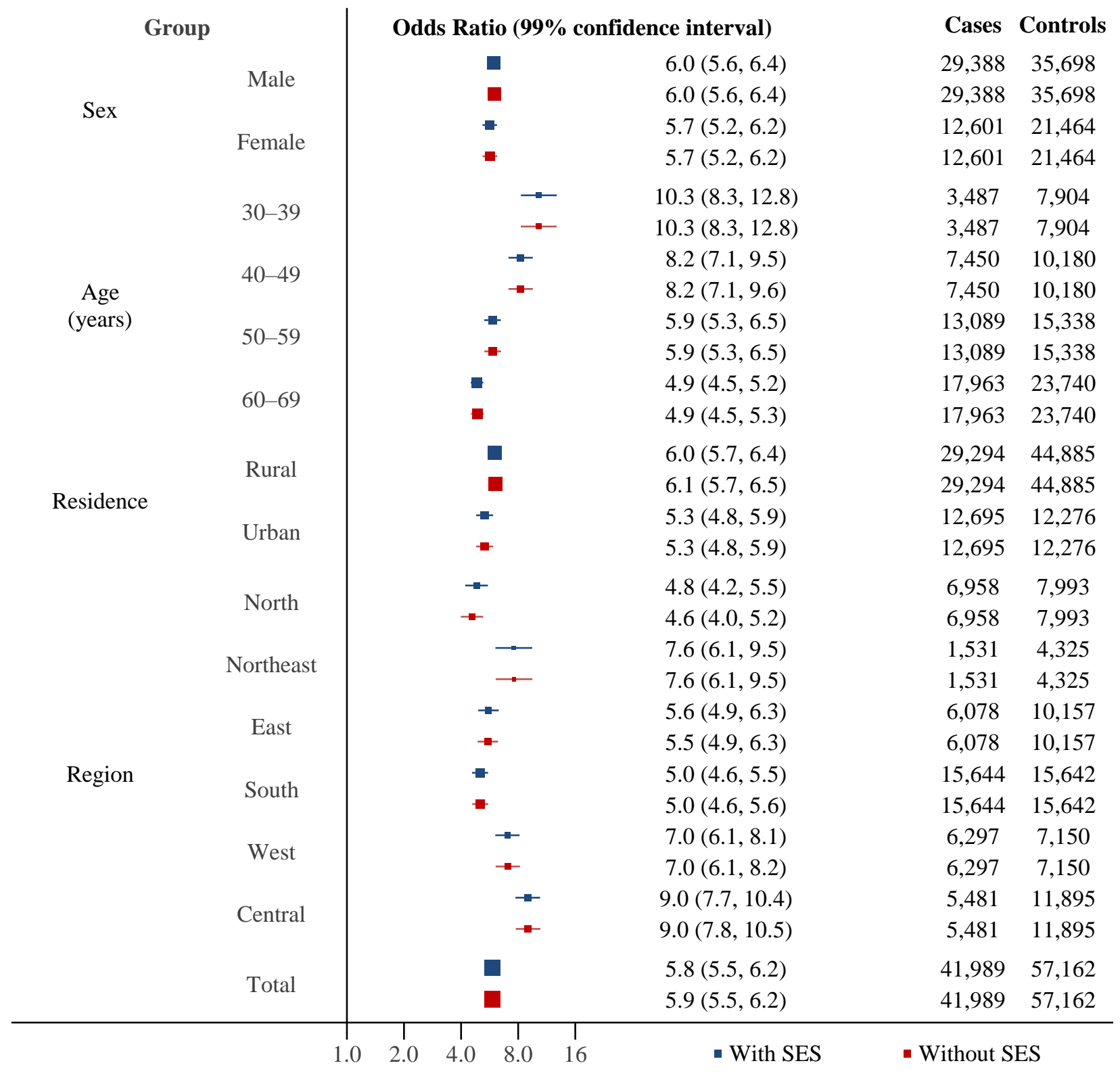




\section{B. Diabetes and Ischemic Heart Disease}

\begin{tabular}{|c|c|c|c|c|c|}
\hline \multicolumn{2}{|c|}{ Group } & \multicolumn{2}{|c|}{ Odds Ratio (99\% confidence interval) } & \multirow{3}{*}{$\begin{array}{c}\text { Cases } \\
29,388 \\
29,388\end{array}$} & \multirow{2}{*}{$\begin{array}{c}\text { Controls } \\
35,698\end{array}$} \\
\hline \multirow{4}{*}{ Sex } & \multirow{2}{*}{ Male } & $=$ & $1.8(1.6,2.0)$ & & \\
\hline & & $=$ & $1.8(1.7,2.0)$ & & 35,698 \\
\hline & \multirow{2}{*}{ Female } & $\rightarrow$ & $2.0(1.7,2.3)$ & 12,601 & 21,464 \\
\hline & & $=$ & $2.0(1.8,2.3)$ & 12,601 & 21,464 \\
\hline \multirow{8}{*}{$\begin{array}{c}\text { Age } \\
\text { (years) }\end{array}$} & \multirow{2}{*}{$30-39$} & $=$ & $1.5(1.0,2.4)$ & 3,487 & 7,904 \\
\hline & & $\longrightarrow$ & $1.5(1.0,2.4)$ & 3,487 & 7,904 \\
\hline & \multirow{2}{*}{$40-49$} & $\rightarrow$ & $1.9(1.5,2.4)$ & 7,450 & 10,180 \\
\hline & & $\rightarrow$ & $1.9(1.5,2.4)$ & 7,450 & 10,180 \\
\hline & \multirow{2}{*}{$50-59$} & $\rightarrow$ & $2.1(1.8,2.4)$ & 13,089 & 15,338 \\
\hline & & $\rightarrow$ & $2.1(1.8,2.4)$ & 13,089 & 15,338 \\
\hline & \multirow{2}{*}{$60-69$} & + & $1.8(1.6,2.0)$ & 17,963 & 23,740 \\
\hline & & $\theta$ & $1.8(1.6,2.1)$ & 17,963 & 23,740 \\
\hline \multirow{4}{*}{ Residence } & \multirow{2}{*}{ Rural } & $=$ & $1.7(1.6,1.9)$ & 29,294 & 44,885 \\
\hline & & 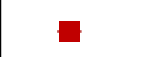 & $1.8(1.6,2.0)$ & 29,294 & 44,885 \\
\hline & \multirow{2}{*}{ Urban } & $\rightarrow$ & $2.1(1.8,2.4)$ & 12,695 & 12,276 \\
\hline & & - & $2.1(1.8,2.5)$ & 12,695 & 12,276 \\
\hline \multirow{14}{*}{ Region } & \multirow{2}{*}{ North } & $\rightarrow$ & $1.8(1.4,2.2)$ & 6,958 & 7,993 \\
\hline & & $\rightarrow$ & $1.9(1.5,2.4)$ & 6,958 & 7,993 \\
\hline & \multirow{2}{*}{ Northeast } &. & $1.7(1.1,2.7)$ & 1,531 & 4,325 \\
\hline & & . & $1.7(1.1,2.7)$ & 1,531 & 4,325 \\
\hline & \multirow{2}{*}{ East } & $\rightarrow$ & $1.3(1.1,1.6)$ & 6,078 & 10,157 \\
\hline & & - & $1.3(1.1,1.6)$ & 6,078 & 10,157 \\
\hline & \multirow{2}{*}{ South } & $\rightarrow$ & $2.0(1.8,2.3)$ & 15,644 & 15,642 \\
\hline & & $=$ & $2.0(1.8,2.3)$ & 15,644 & 15,642 \\
\hline & \multirow{2}{*}{ West } & $\rightarrow$ & $2.8(2.2,3.5)$ & 6,297 & 7,150 \\
\hline & & $\rightarrow$ & $2.8(2.2,3.5)$ & 6,297 & 7,150 \\
\hline & \multirow{2}{*}{ Central } & $\longrightarrow$ & $2.1(1.5,3.0)$ & 5,481 & 11,895 \\
\hline & & $\longrightarrow$ & $2.1(1.5,3.0)$ & 5,481 & 11,895 \\
\hline & \multirow{2}{*}{ Total } & $\square$ & $1.9(1.7,2.0)$ & 41,989 & 57,162 \\
\hline & & 口 & $1.9(1.7,2.0)$ & 41,989 & 57,162 \\
\hline
\end{tabular}


eFigure 13 Total and stratified estimates of the association between (A) hypertension or (B) diabetes and stroke mortality in high-burden states, with and without socioeconomic status (SES) adjustment (2001 to 2013 only). Estimates are adjusted for age, sex, rurality, region, smoking, alcohol use, and year. The area of each box is proportional to the sample size (cases and controls).

\section{A. Hypertension and Stroke, High-Burden}

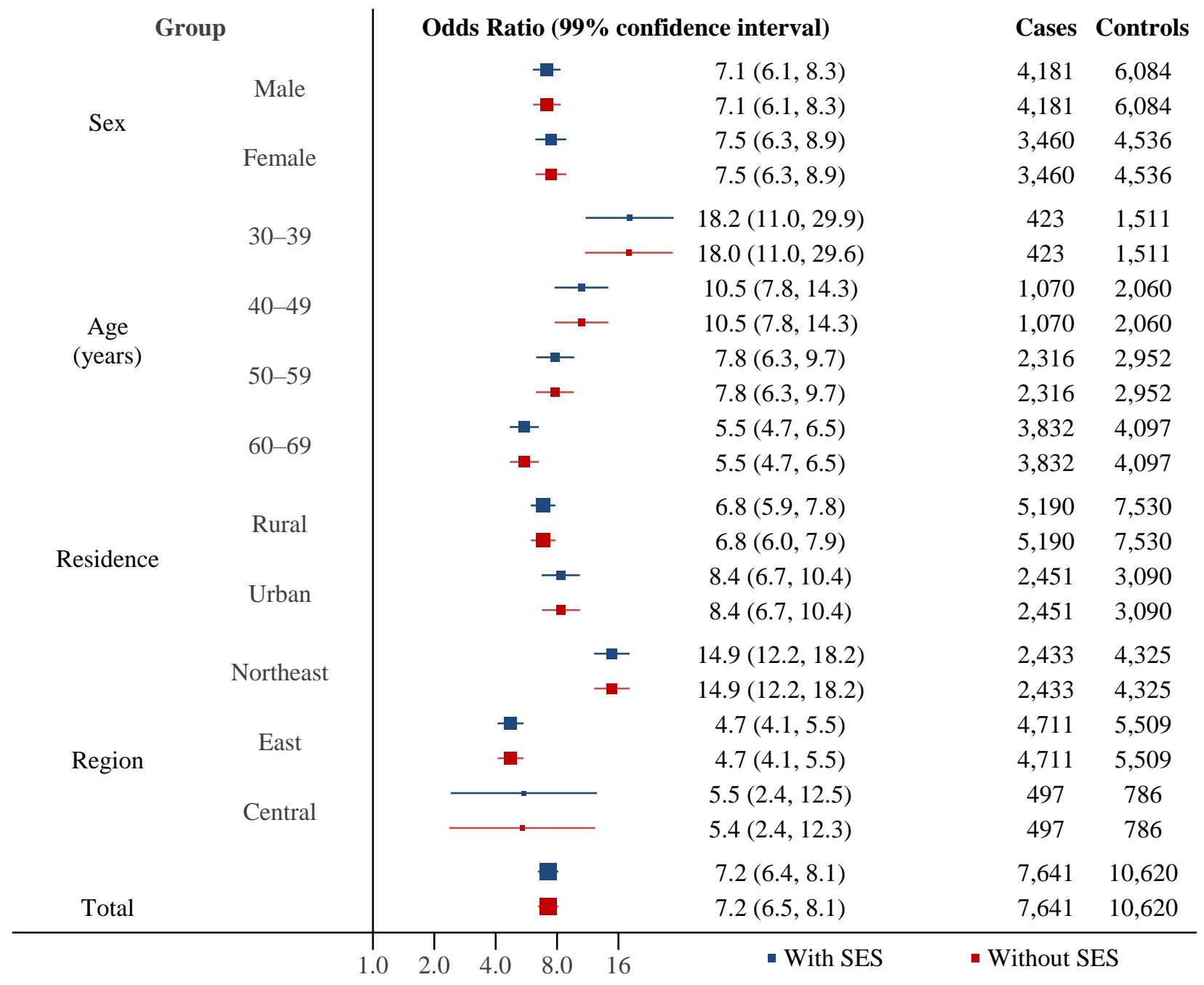




\section{B. Diabetes and Stroke, High-Burden}

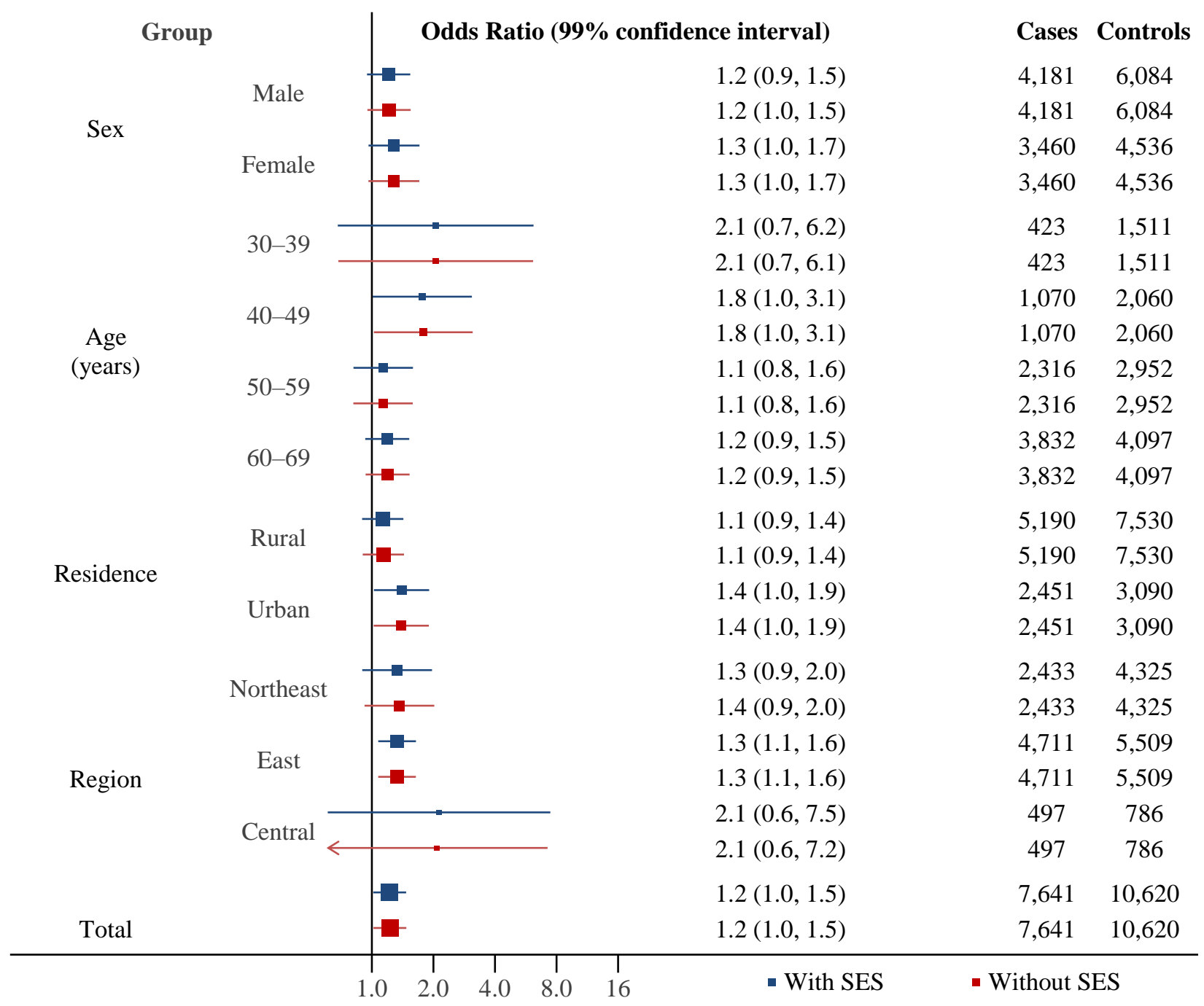


eFigure 14 Total and stratified estimates of the association between (A) hypertension or (B) diabetes and stroke mortality in low-burden states, with and without socioeconomic status (SES) adjustment (2001 to 2013 only). Estimates are adjusted for age, sex, rurality, region, smoking, alcohol use, and year. The area of each box is proportional to the sample size (cases and controls).

\section{A. Hypertension and Stroke, Low-Burden}

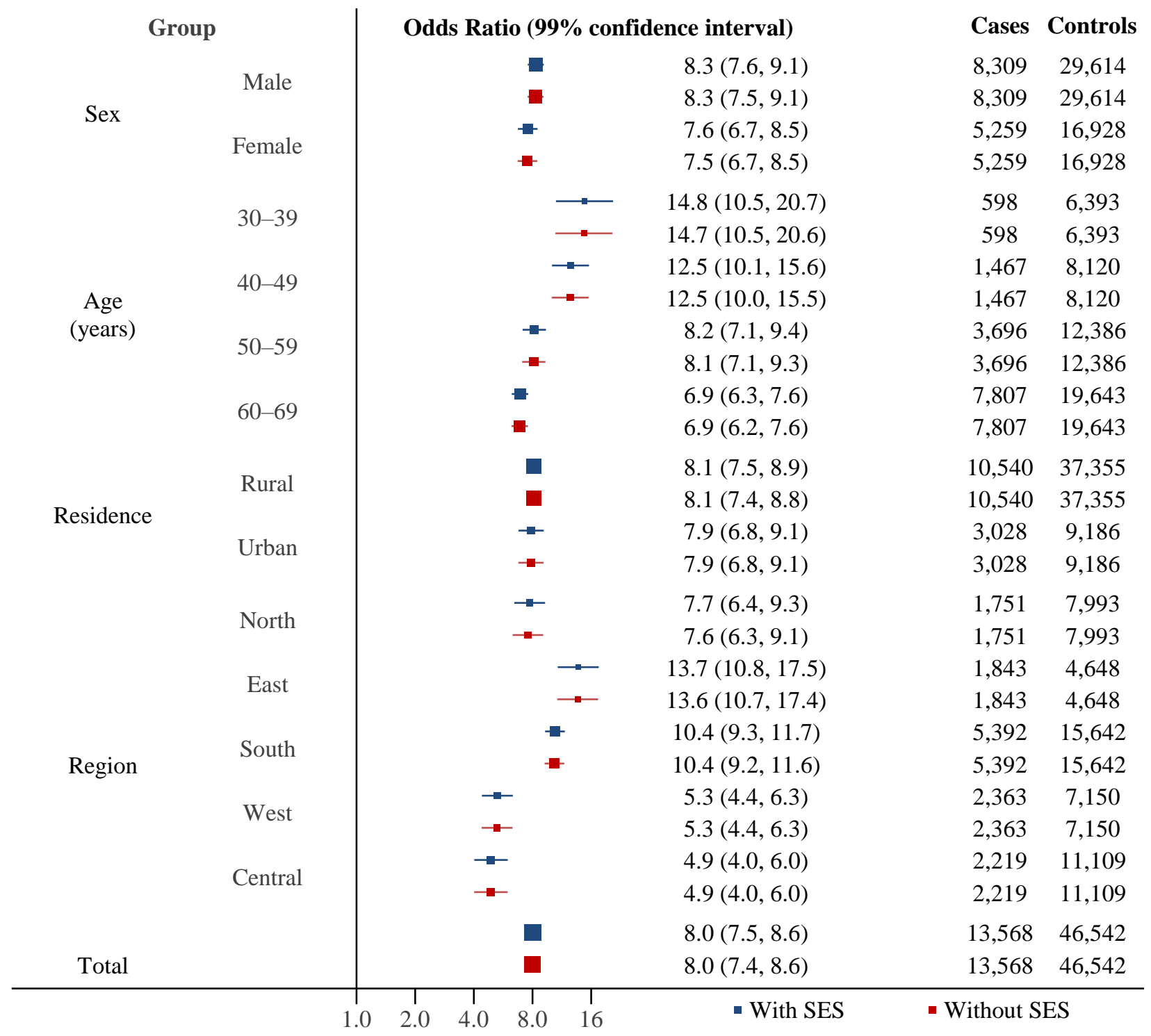




\section{B. Diabetes and Stroke, Low-Burden}

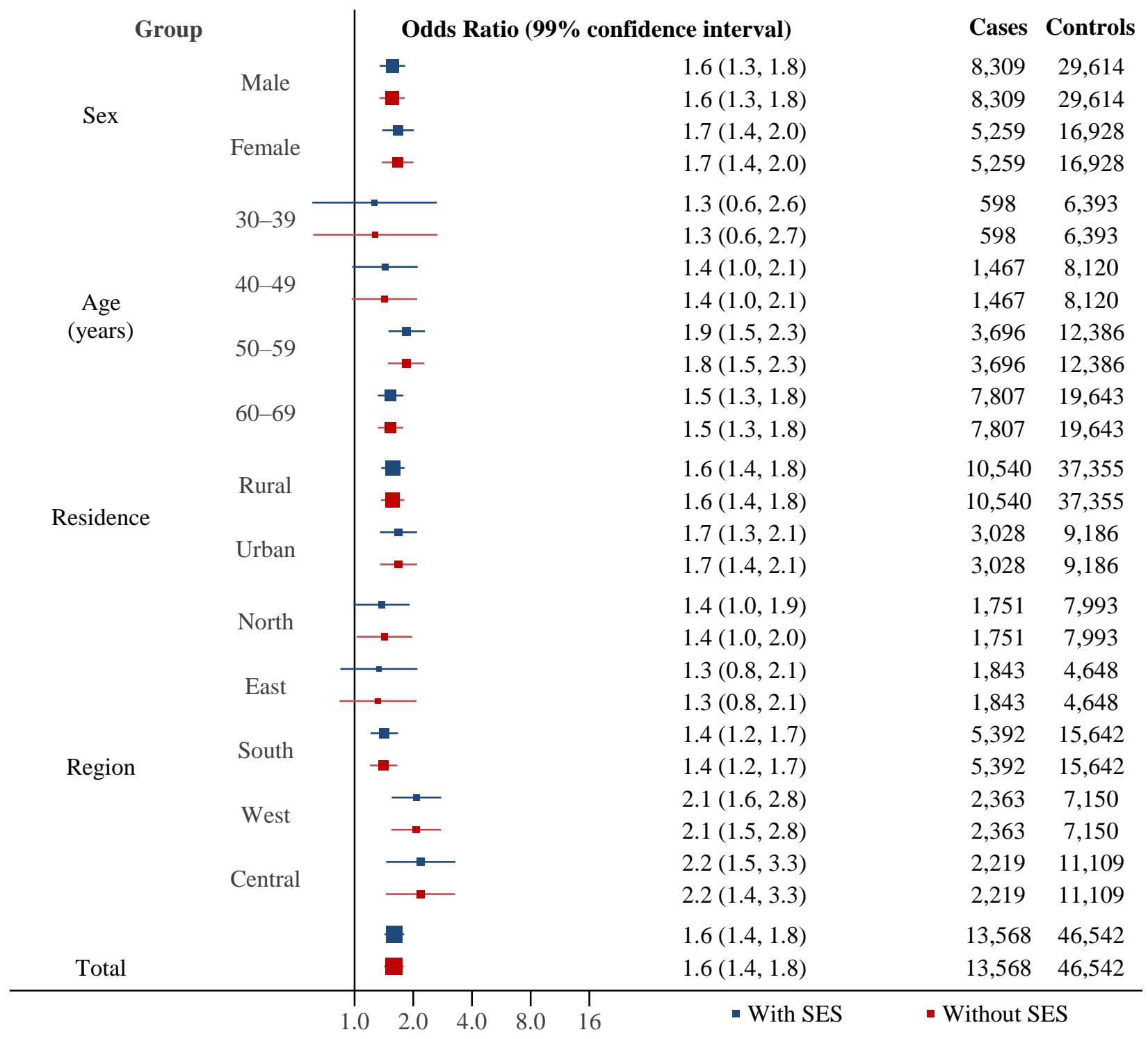


eFigure 15 Total and stratified estimates of the association between (A) hypertension or (B) diabetes and ischemic heart disease mortality, with and without adjustment for clustering at the district level (2001 to 2013 only). Estimates are adjusted for age, sex, rurality, region, smoking, alcohol use, and year. The area of each box is proportional to the sample size (cases and controls).

\section{A. Hypertension and Ischemic Heart Disease}

\begin{tabular}{|c|c|c|c|c|c|}
\hline \multicolumn{2}{|c|}{ Group } & \multicolumn{2}{|c|}{ Odds Ratio ( $99 \%$ confidence interval) } & Cases & Controls \\
\hline \multirow{4}{*}{ Sex } & \multirow{2}{*}{ Male } & - & $6.0(5.6,6.4)$ & 29,388 & 35,698 \\
\hline & & - & $6.0(5.6,6.4)$ & 29,388 & 35,698 \\
\hline & \multirow{2}{*}{ Female } & 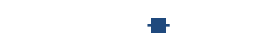 & $5.7(5.2,6.2)$ & 12,601 & 21,464 \\
\hline & & 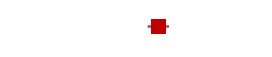 & $5.7(5.2,6.2)$ & 12,601 & 21,464 \\
\hline \multirow{8}{*}{$\begin{array}{c}\text { Age } \\
\text { (years) }\end{array}$} & \multirow{2}{*}{ 30-39 } & $\rightarrow$ & $10.3(8.2,12.8)$ & 3,487 & 7,904 \\
\hline & & $\rightarrow-$ & $10.3(8.3,12.8)$ & 3,487 & 7,904 \\
\hline & \multirow{2}{*}{$40-49$} & $\rightarrow$ & $8.2(7.1,9.5)$ & 7,450 & 10,180 \\
\hline & & $\rightarrow$ & $8.2(7.1,9.6)$ & 7,450 & 10,180 \\
\hline & \multirow{2}{*}{$50-59$} & 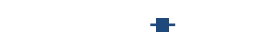 & $5.9(5.3,6.5)$ & 13,089 & 15,338 \\
\hline & & 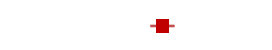 & $5.9(5.3,6.5)$ & 13,089 & 15,338 \\
\hline & \multirow{2}{*}{$60-69$} & $=$ & $4.9(4.5,5.3)$ & 17,963 & 23,740 \\
\hline & & $=$ & $4.9(4.5,5.3)$ & 17,963 & 23,740 \\
\hline \multirow{4}{*}{ Residence } & \multirow{2}{*}{ Rural } & - & $6.1(5.6,6.5)$ & 29,294 & 44,885 \\
\hline & & - & $6.1(5.7,6.5)$ & 29,294 & 44,885 \\
\hline & \multirow{2}{*}{ Urban } & - & $5.3(4.8,5.9)$ & 12,695 & 12,276 \\
\hline & & $=$ & $5.3(4.8,5.9)$ & 12,695 & 12,276 \\
\hline \multirow{14}{*}{ Region } & \multirow{2}{*}{ North } & $\rightarrow$ & $4.6(4.0,5.3)$ & 6,958 & 7,993 \\
\hline & & - & $4.6(4.0,5.2)$ & 6,958 & 7,993 \\
\hline & \multirow{2}{*}{ Northeast } & $\rightarrow$ & $7.5(6.0,9.5)$ & 1,531 & 4,325 \\
\hline & & $\rightarrow$ & $7.6(6.1,9.5)$ & 1,531 & 4,325 \\
\hline & \multirow{2}{*}{ East } & $\rightarrow$ & $5.6(4.9,6.3)$ & 6,078 & 10,157 \\
\hline & & - & $5.5(4.9,6.3)$ & 6,078 & 10,157 \\
\hline & \multirow{2}{*}{ South } & $=$ & $5.0(4.5,5.6)$ & 15,644 & 15,642 \\
\hline & & $=$ & $5.0(4.6,5.6)$ & 15,644 & 15,642 \\
\hline & \multirow{2}{*}{ West } & $\rightarrow$ & $7.1(6.0,8.4)$ & 6,297 & 7,150 \\
\hline & & - & $7.0(6.1,8.2)$ & 6,297 & 7,150 \\
\hline & \multirow{2}{*}{ Central } & $\rightarrow$ & $9.1(7.7,10.7)$ & 5,481 & 11,895 \\
\hline & & - & $9.0(7.8,10.5)$ & 5,481 & 11,895 \\
\hline & \multirow{2}{*}{ Total } & $\mathbf{\square}$ & $5.8(5.5,6.2)$ & 41,989 & 57,162 \\
\hline & & $\square$ & $5.9(5.5,6.2)$ & 41,989 & 57,162 \\
\hline
\end{tabular}




\section{B. Diabetes and Ischemic Heart Disease}

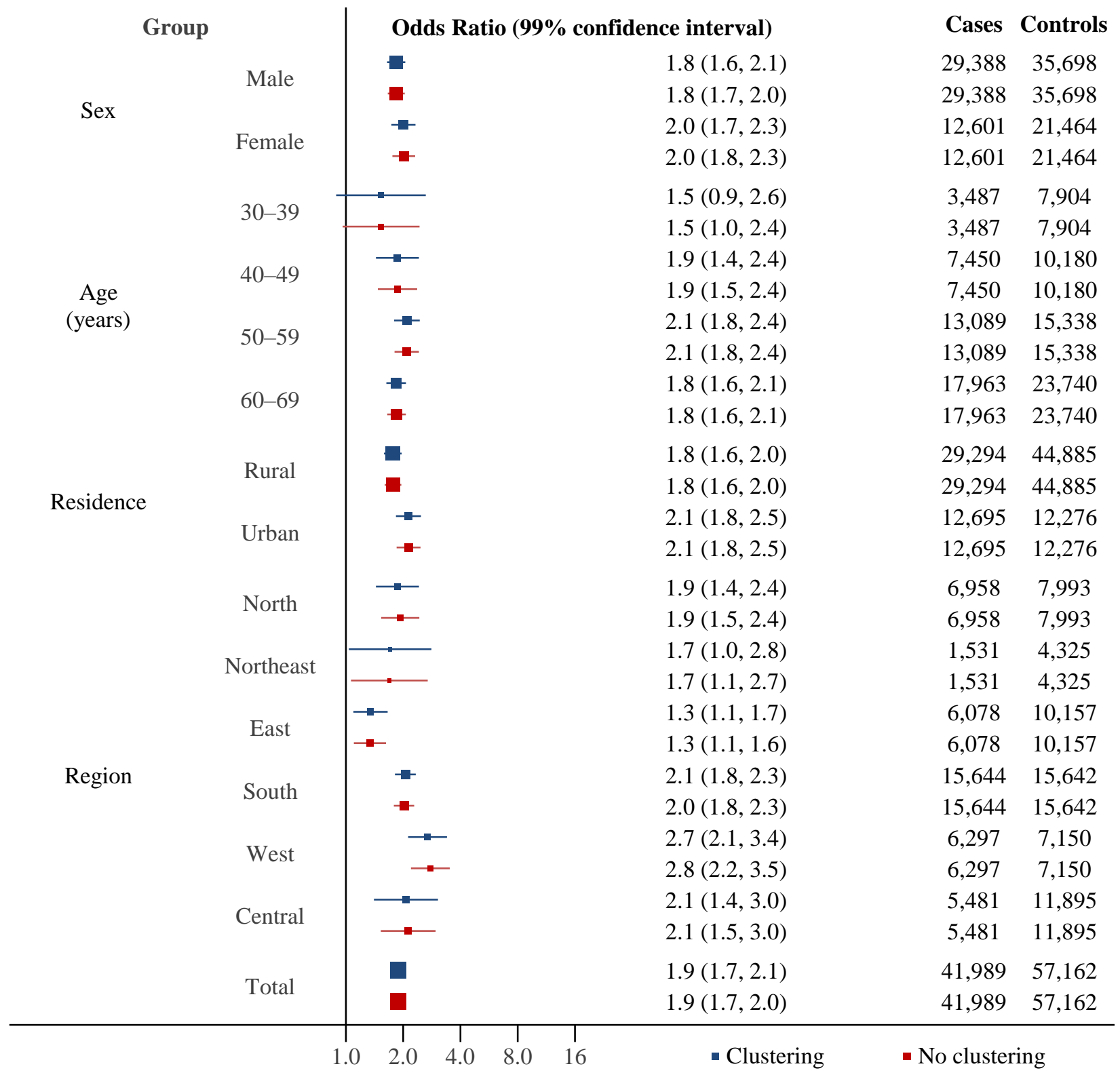


eFigure 16 Total and stratified estimates of the association between (A) hypertension or (B) diabetes and stroke mortality in high-burden states, with and without adjustment for clustering at the district level (2001 to 2013 only). Estimates are adjusted for age, sex, rurality, region, smoking, alcohol use, and year. The area of each box is proportional to the sample size (cases and controls).

\section{A. Hypertension and Stroke, High-Burden}

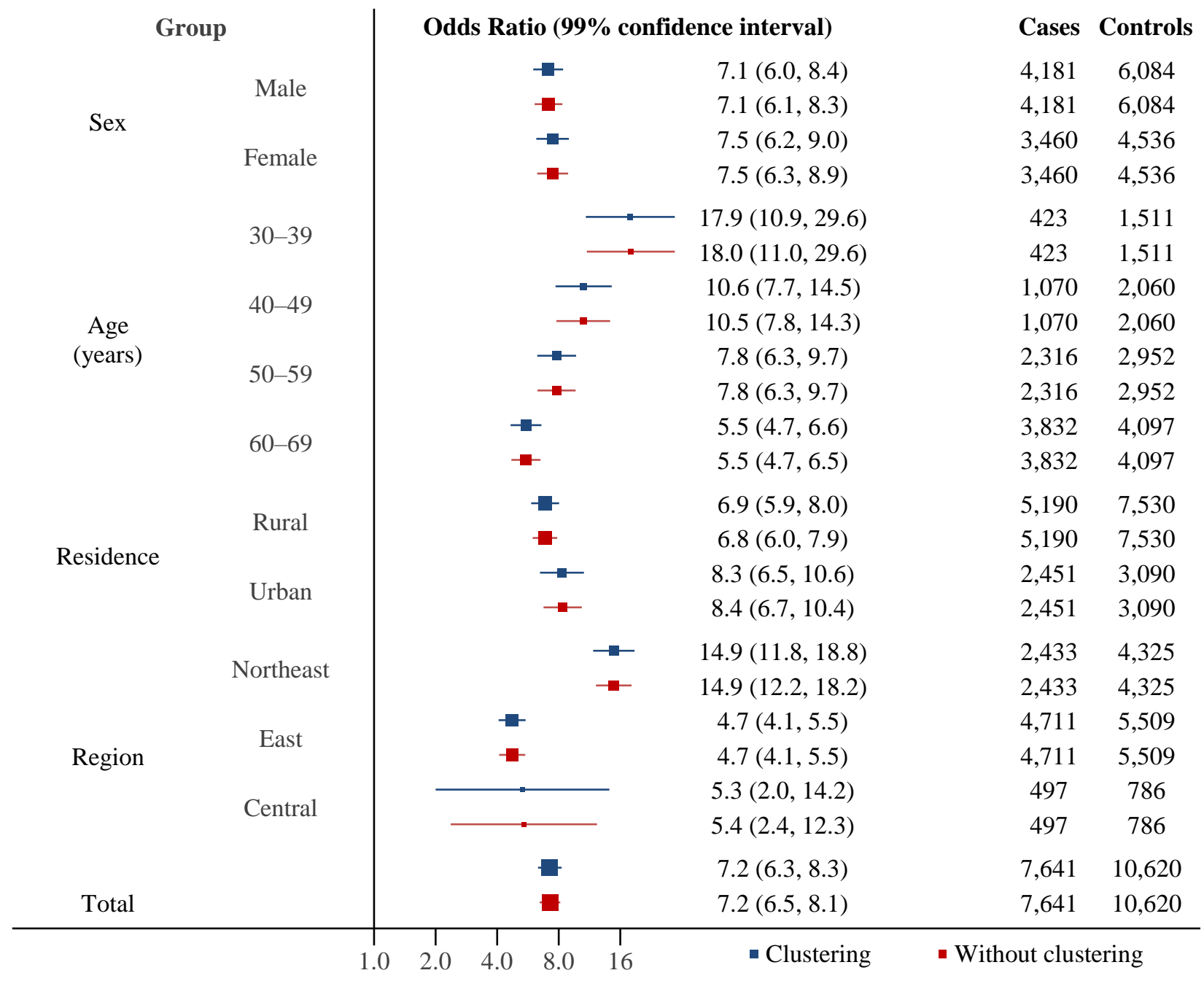




\section{B. Diabetes and Stroke, High-Burden}

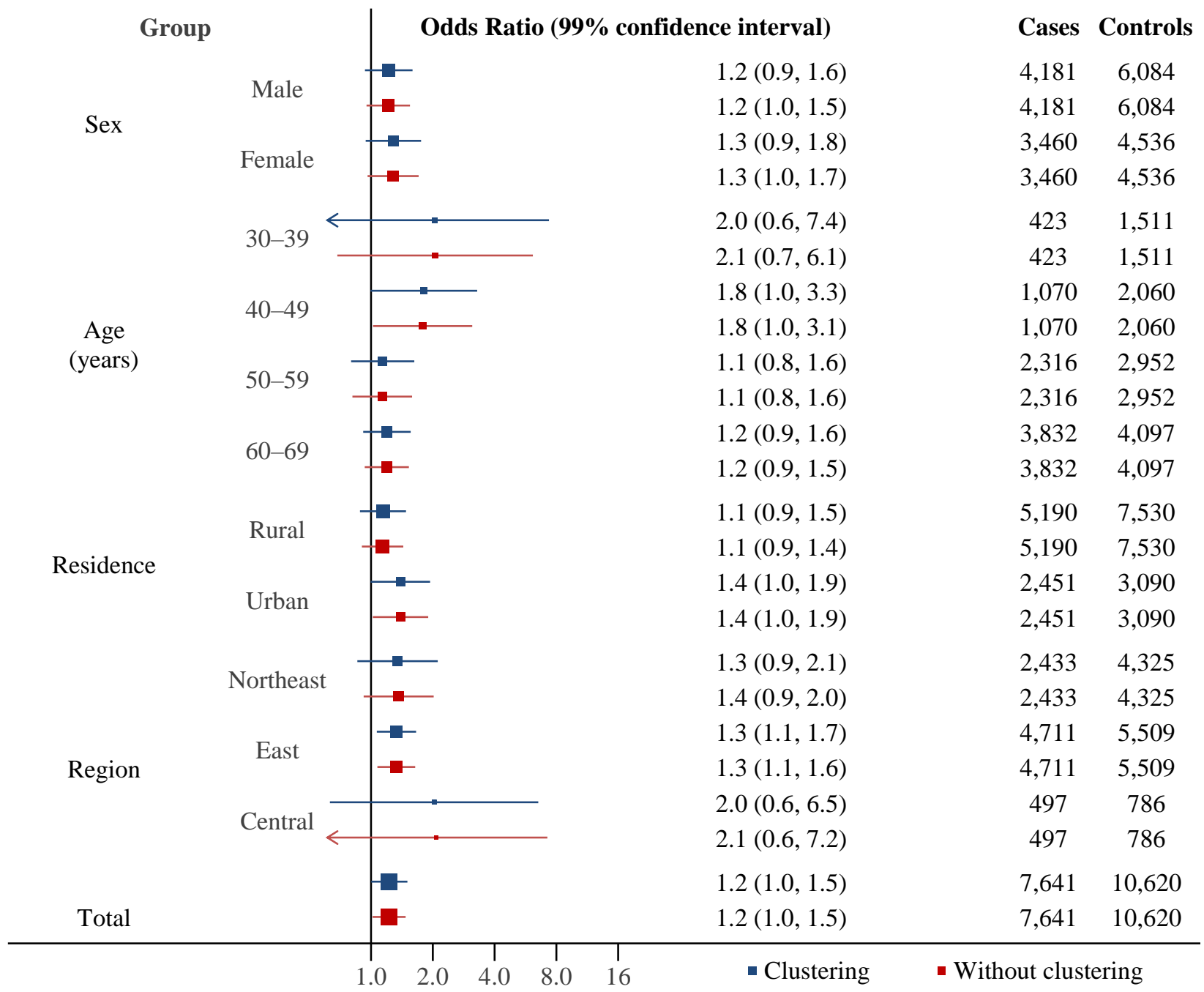


eFigure 17 Total and stratified estimates of the association between (A) hypertension or (B) diabetes and stroke mortality in low-burden states, with and without adjustment for clustering at the district level (2001 to 2013 only). Estimates are adjusted for age, sex, rurality, region, smoking, alcohol use, and year. The area of each box is proportional to the sample size (cases and controls).

\section{A. Hypertension and Stroke, Low-Burden}

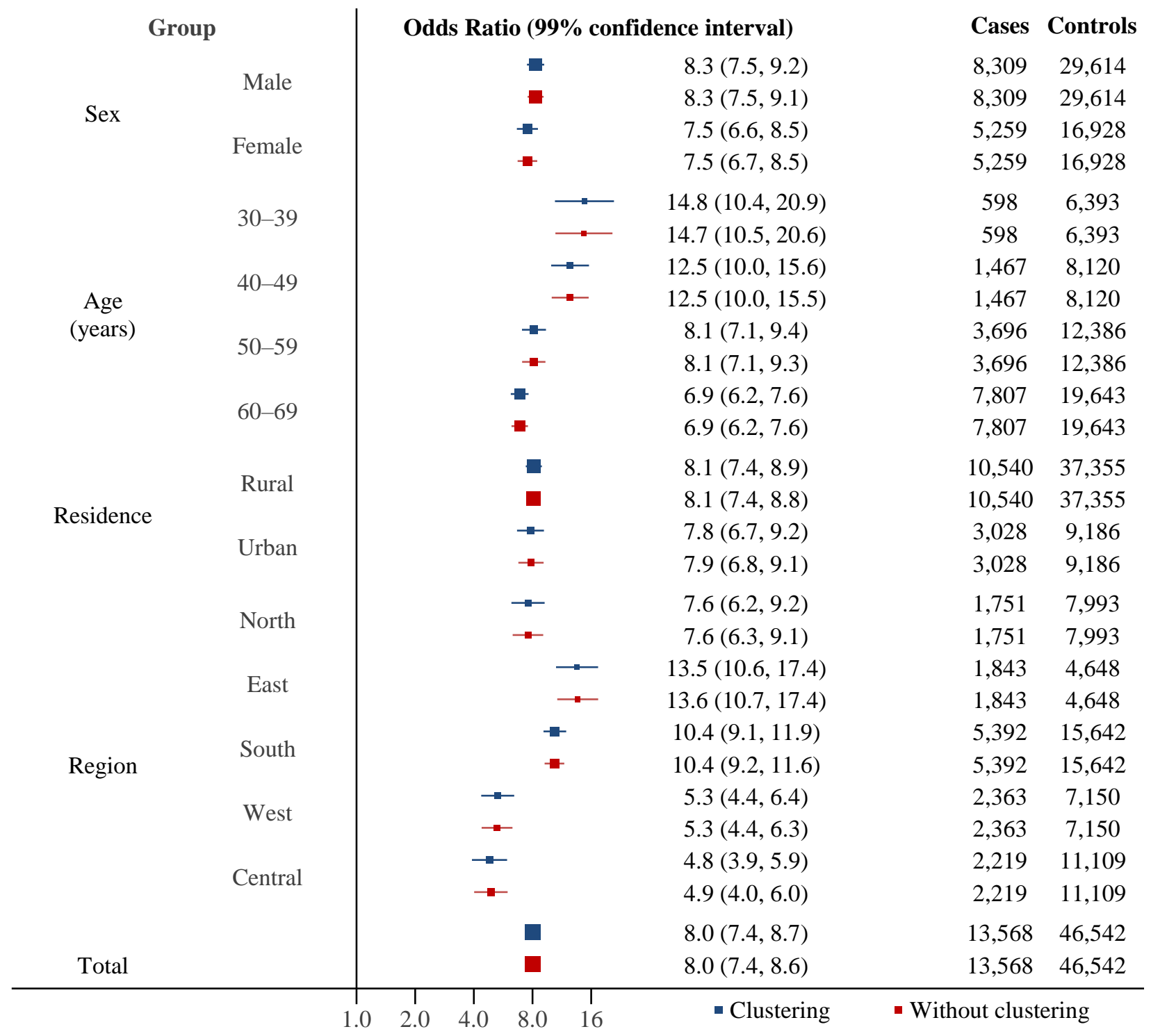




\section{B. Diabetes and Stroke, Low-Burden}

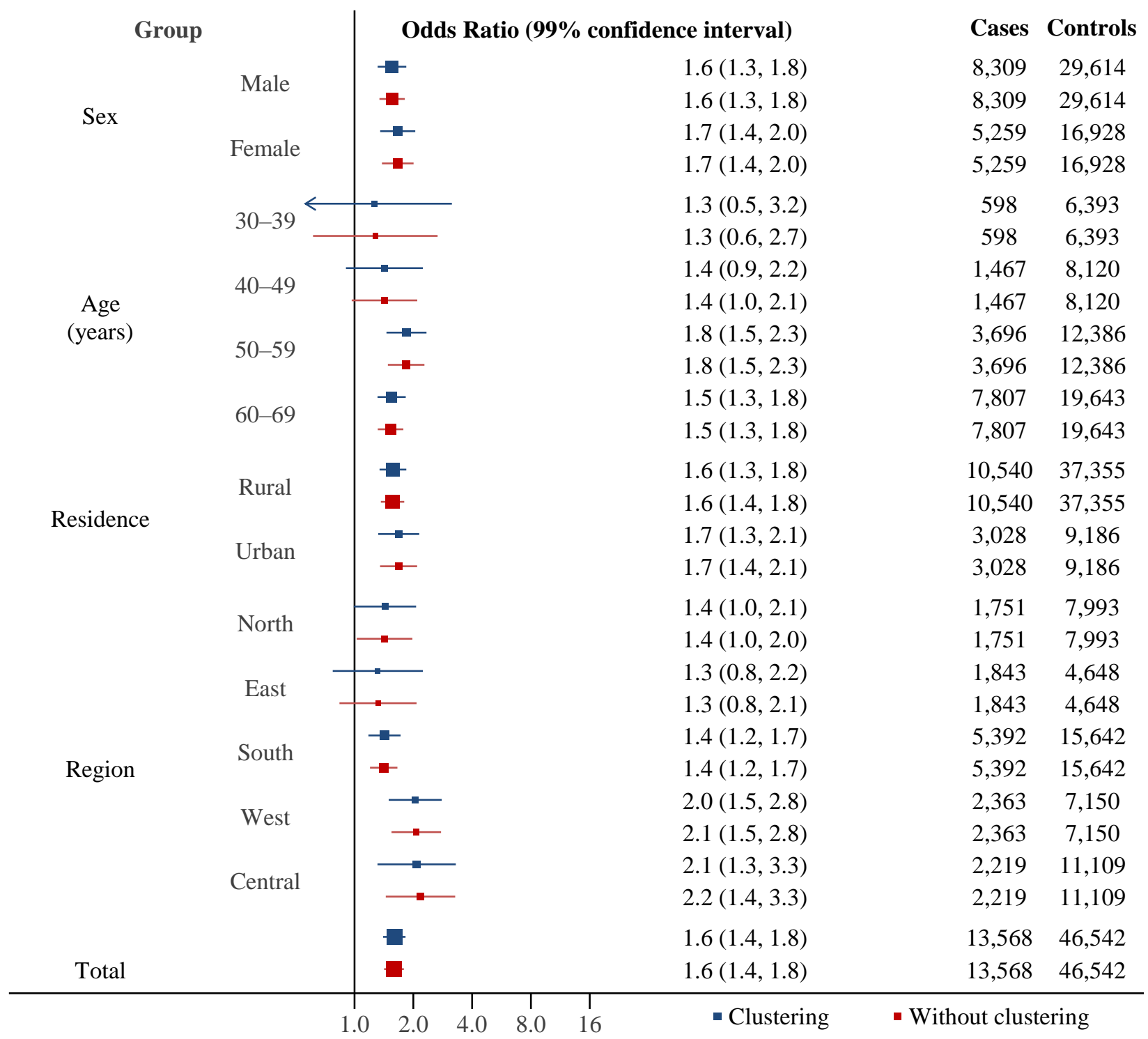


eFigure 18 Additional analysis using logistic regression to estimate the association between respondent-reported any medication usage (medication names unavailable) and mortality from ischemic heart disease and stroke among people with hypertension alone, diabetes alone, or both. Data are for 2004 onward (no available medication data for 2001-03). Estimates are adjusted for age, sex, urban or rural residence, region, smoking, alcohol use, and year. The area of each box is proportional to the sample size (cases and controls).

\begin{tabular}{|c|c|c|c|c|c|}
\hline Outcome & Risk Factor(s) & Odds Ratio ( $99 \%$ co & e interval) & Cases & Controls \\
\hline \multirow{3}{*}{$\begin{array}{l}\text { Ischemic Heart } \\
\text { Disease }\end{array}$} & Hypertension Alone & & $0.6(0.5,0.6)$ & 9,529 & 2,648 \\
\hline & Diabetes Alone & & $1.1(0.8,1.5)$ & 1,487 & 861 \\
\hline & Hypertension and Diabetes & & $0.7(0.5,0.8)$ & 3,637 & 857 \\
\hline \multirow{3}{*}{$\begin{array}{c}\text { Stroke } \\
\text { (high-burden) }\end{array}$} & Hypertension Alone & & $0.7(0.5,1.0)$ & 2,145 & 661 \\
\hline & Diabetes Alone & & $0.8(0.4,1.8)$ & 176 & 166 \\
\hline & Hypertension and Diabetes & $\square$ & $0.4(0.2,0.7)$ & 646 & 201 \\
\hline \multirow{3}{*}{$\begin{array}{c}\text { Stroke } \\
\text { (low-burden) }\end{array}$} & Hypertension Alone & & $0.9(0.7,1.0)$ & 3,304 & 1,987 \\
\hline & Diabetes Alone & & $0.7(0.5,1.1)$ & 408 & 695 \\
\hline & Hypertension and Diabetes & 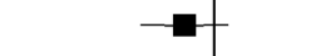 & $0.8(0.6,1.1)$ & 1,058 & 656 \\
\hline
\end{tabular}


eTable 4 Sensitivity and additional analyses of the association between respondent-reported medication use and ischemic heart disease or stroke mortality among people with hypertension alone, diabetes alone, or both hypertension and diabetes. Results pertain to India as a whole; findings by state could not be generated due to sample size limitations.

\begin{tabular}{|c|c|c|c|c|c|c|c|c|c|c|c|c|}
\hline & \multicolumn{11}{|c|}{ Odds Ratios ( $99 \%$ confidence interval) } \\
\hline & & $\begin{array}{l}\text { Main } \\
\text { Estimate }\end{array}$ & Men & Women & $\begin{array}{c}\text { Initial } \\
\text { Agreement }\end{array}$ & $\begin{array}{l}\text { Prior Heart } \\
\text { Disease }\end{array}$ & $\begin{array}{c}\text { No Prior } \\
\text { Heart } \\
\text { Disease }\end{array}$ & $\begin{array}{l}\text { Injury } \\
\text { Controls }\end{array}$ & $\begin{array}{l}\text { Cancer } \\
\text { Controls }\end{array}$ & $\begin{array}{l}\text { Respiratory } \\
\text { Controls }\end{array}$ & $\begin{array}{c}\text { SES } \\
\text { Adjustment }\end{array}$ & $\begin{array}{l}\text { Clustering } \\
\text { Adjustment }\end{array}$ \\
\hline \multirow{3}{*}{$\begin{array}{l}\text { Ischemic } \\
\text { Heart } \\
\text { Disease }\end{array}$} & Hypertension Alone & $\begin{array}{l}0.6(0.5, \\
0.6)\end{array}$ & $\begin{array}{l}0.5(0.5 \\
0.6)\end{array}$ & $\begin{array}{l}0.6(0.5 \\
0.7) \\
\end{array}$ & $\begin{array}{l}0.6(0.5, \\
0.6)\end{array}$ & $\begin{array}{l}0.6(0.4, \\
0.8)\end{array}$ & $\begin{array}{l}0.6(0.5, \\
0.7) \\
\end{array}$ & $\begin{array}{l}1.5(1.1, \\
2.3)\end{array}$ & $\begin{array}{l}0.4(0.3, \\
0.5)\end{array}$ & $\begin{array}{l}0.6(0.5, \\
0.7) \\
\end{array}$ & $\begin{array}{l}0.6(0.5, \\
0.6)\end{array}$ & $\begin{array}{l}0.6(0.5, \\
0.7)\end{array}$ \\
\hline & Diabetes Alone & $\begin{array}{l}1.1(0.8, \\
1.5)\end{array}$ & $\begin{array}{l}1.2(0.8, \\
1.7)\end{array}$ & N/A & $\begin{array}{l}1.1(0.8, \\
1.5)\end{array}$ & $\begin{array}{l}0.8(0.3, \\
2.1)\end{array}$ & $\begin{array}{l}1.1(0.8, \\
1.5)\end{array}$ & $\begin{array}{l}2.3(1.3 \\
4.4) \\
\end{array}$ & $\begin{array}{l}0.8(0.5, \\
1.2)\end{array}$ & $\begin{array}{l}1.2(0.8, \\
1.8)\end{array}$ & $\begin{array}{l}1.1(0.8, \\
1.5)\end{array}$ & $\begin{array}{l}1.2(0.8, \\
1.6)\end{array}$ \\
\hline & $\begin{array}{l}\text { Hypertension and } \\
\text { Diabetes }\end{array}$ & $\begin{array}{l}0.7(0.5 \\
0.8)\end{array}$ & $\begin{array}{l}0.7(0.5, \\
0.9)\end{array}$ & N/A & $\begin{array}{l}0.6(0.5, \\
0.8)\end{array}$ & $\begin{array}{l}0.7(0.4, \\
1.2)\end{array}$ & $\begin{array}{l}0.7(0.5, \\
0.9)\end{array}$ & $\begin{array}{l}1.3(0.7, \\
2.5)\end{array}$ & $\begin{array}{l}0.4(0.3, \\
0.6) \\
\end{array}$ & $\begin{array}{l}0.8(0.6 \\
1.2)\end{array}$ & $\begin{array}{l}0.7(0.5, \\
0.9)\end{array}$ & $\begin{array}{l}0.7(0.5, \\
0.9)\end{array}$ \\
\hline \multirow{3}{*}{$\begin{array}{l}\text { Stroke } \\
\text { (high- } \\
\text { burden) }\end{array}$} & Hypertension Alone & $\begin{array}{l}0.7(0.5 \\
1.0)\end{array}$ & $\begin{array}{l}0.9(0.6 \\
1.4)\end{array}$ & $\begin{array}{l}0.6(0.4, \\
0.9)\end{array}$ & $\begin{array}{l}0.7(0.5, \\
0.9)\end{array}$ & $\begin{array}{l}0.7(0.2, \\
2.2)\end{array}$ & $\begin{array}{l}0.6(0.4, \\
0.8)\end{array}$ & $\begin{array}{l}2.2(0.9, \\
5.4)\end{array}$ & $\begin{array}{l}0.6(0.4, \\
0.9)\end{array}$ & $\begin{array}{l}0.6(0.4, \\
1.0)\end{array}$ & $\begin{array}{l}0.7(0.5, \\
1.0)\end{array}$ & $\begin{array}{l}0.7(0.5, \\
1.0)\end{array}$ \\
\hline & Diabetes Alone & $\begin{array}{l}0.8(0.4, \\
1.8)\end{array}$ & $\begin{array}{l}0.8(0.3, \\
2.2)\end{array}$ & $\begin{array}{l}0.6(0.2, \\
2.5)\end{array}$ & $\begin{array}{l}1.1(0.5, \\
2.4)\end{array}$ & N/A & $\begin{array}{l}1.2(0.4, \\
3.5)\end{array}$ & N/A & $\begin{array}{l}0.5(0.2, \\
1.3)\end{array}$ & N/A & $\begin{array}{l}0.9(0.4, \\
2.0)\end{array}$ & $\begin{array}{l}0.9(0.4, \\
2.0)\end{array}$ \\
\hline & $\begin{array}{l}\text { Hypertension and } \\
\text { Diabetes }\end{array}$ & $\begin{array}{l}0.4(0.2, \\
0.7)\end{array}$ & $\begin{array}{l}0.5(0.2, \\
1.0)\end{array}$ & $\begin{array}{l}0.2(0.1, \\
0.6)\end{array}$ & $\begin{array}{l}0.4(0.2, \\
0.6) \\
\end{array}$ & N/A & $\begin{array}{l}0.2(0.1, \\
0.5)\end{array}$ & N/A & $\begin{array}{l}0.2(0.1, \\
0.4)\end{array}$ & N/A & $\begin{array}{l}0.4(0.2, \\
0.7)\end{array}$ & $\begin{array}{l}0.4(0.2, \\
0.7)\end{array}$ \\
\hline \multirow{3}{*}{$\begin{array}{l}\text { Stroke } \\
\text { (low- } \\
\text { burden) }\end{array}$} & Hypertension Alone & $\begin{array}{l}0.9(0.7, \\
1.0) \\
\end{array}$ & $\begin{array}{l}0.8(0.7, \\
1.0) \\
\end{array}$ & $\begin{array}{l}0.9(0.7, \\
1.2) \\
\end{array}$ & $\begin{array}{l}0.9(0.7, \\
1.1) \\
\end{array}$ & $\begin{array}{l}1.1(0.5, \\
2.3) \\
\end{array}$ & $\begin{array}{l}0.7(0.6, \\
0.9)\end{array}$ & $\begin{array}{l}2.2(1.4, \\
3.4) \\
\end{array}$ & $\begin{array}{l}0.6(0.5, \\
0.8) \\
\end{array}$ & $\begin{array}{l}0.9(0.7, \\
1.1) \\
\end{array}$ & $\begin{array}{l}0.8(0.7, \\
1.0) \\
\end{array}$ & $\begin{array}{l}0.8(0.7, \\
1.0) \\
\end{array}$ \\
\hline & Diabetes Alone & $\begin{array}{l}0.7(0.5, \\
1.1) \\
\end{array}$ & $\begin{array}{l}0.9(0.5, \\
1.5) \\
\end{array}$ & $\begin{array}{l}0.5(0.3, \\
1.0) \\
\end{array}$ & $\begin{array}{l}0.8(0.5, \\
1.2) \\
\end{array}$ & N/A & $\begin{array}{l}0.7(0.4, \\
1.1) \\
\end{array}$ & $\begin{array}{l}1.9(0.9, \\
3.9) \\
\end{array}$ & $\begin{array}{l}0.5(0.3, \\
0.9) \\
\end{array}$ & $\begin{array}{l}0.7(0.4, \\
1.2) \\
\end{array}$ & $\begin{array}{l}0.8(0.5, \\
1.2) \\
\end{array}$ & $\begin{array}{l}0.8(0.5, \\
1.2) \\
\end{array}$ \\
\hline & $\begin{array}{l}\text { Hypertension and } \\
\text { Diabetes }\end{array}$ & $\begin{array}{l}0.8(0.6 \\
1.1) \\
\end{array}$ & $\begin{array}{l}0.7(0.4, \\
1.1) \\
\end{array}$ & $\mathrm{N} / \mathrm{A}$ & $\begin{array}{l}0.8(0.5, \\
1.1) \\
\end{array}$ & $\begin{array}{l}0.9(0.3, \\
2.7)\end{array}$ & $\begin{array}{l}0.6(0.4, \\
1.0)\end{array}$ & $\begin{array}{l}1.8(0.8, \\
3.9) \\
\end{array}$ & $\begin{array}{l}0.6(0.4, \\
0.9)\end{array}$ & $\begin{array}{l}0.8(0.5, \\
1.3)\end{array}$ & $\begin{array}{l}0.8(0.6, \\
1.2)\end{array}$ & $\begin{array}{l}0.8(0.6, \\
1.2)\end{array}$ \\
\hline
\end{tabular}

Abbreviations: N/A, result could not be estimated due to sample size limitations 


\section{Appendix References}

1. Kumar R, Thakur JS, Rao BT, Singh MMC, Bhatia SPS. Validity of verbal autopsy in determining causes of adult deaths. Indian J Public Health. 2006;50(2):90-94.

2. Ke C, Gupta R, Xavier D, et al. Divergent trends in ischaemic heart disease and stroke mortality in India from 2000 to 2015: a nationally representative mortality study. The Lancet Global Health. 2018;6(8):e914-e923. doi:10.1016/S2214-109X(18)30242-0 